SUPPORTING INFORMATION

\title{
UiO-66 Metal-Organic Framework as an Anode for a Potassium-Ion Battery: Quantum Mechanical Analysis
}

\author{
Anwen Tang, ${ }^{a}$ Xiaojie He, ${ }^{\text {a }}$ Huimin Yin, ${ }^{\text {a }}$ Yi Li, ${ }^{\text {a }}$ Yongfan Zhang, ${ }^{\text {a }}$
}

Shuping Huang, *,a,b Donald G. Truhlar*,c,d

${ }^{a}$ College of Chemistry, Fuzhou University, Fuzhou, 350002 Fujian, China.

bFujian Provincial Key Laboratory of Electrochemical Energy Storage Materials, Fuzhou, 350002 Fujian, China.

'Department of Chemistry, 207 Pleasant Street SE, University of Minnesota, Minneapolis, MN 55455-0431, USA

${ }^{\mathrm{d} C h e m i c a l}$ Theory Center and Minnesota Supercomputing Institute, 207 Pleasant Street SE, University of Minnesota, Minneapolis, MN 55455-0431, USA

*E-mail: huangshp@gmail.com,truhlar@umn.edu

\section{Table of contents}

$\begin{array}{lc}\text { Tables S1 to S3 } & \text { page } \\ \text { Tables S4 to S6 } & \text { S-2 } \\ \text { Tables S7 and S8 } & \text { S-4 } \\ \text { Table S9 } & \text { S-5 } \\ \text { Figure S1 } & \text { S-6 } \\ \text { Figures S2 and S3 } & \text { S-7 } \\ \text { Figure S4 } & \text { S-8 } \\ \text { Figure S5 } & \text { S-10 } \\ \text { Structure data } & \text { S-12 }\end{array}$


Table $\mathrm{S} 1 \mathrm{The} \mathrm{CM} 5$ charges of $\mathrm{K}$ at the transition state along various paths.

\begin{tabular}{cccccc}
\hline Path & $\mathbf{1}$ & $\mathbf{2}$ & $\mathbf{3}$ & $\mathbf{4}$ & $\mathbf{5}$ \\
\hline K charge & 0.82 & 0.76 & 0.81 & 0.77 & 0.77 \\
\hline
\end{tabular}

Table S2. Energies of binding (eV) and average M-C $(\mathrm{M}=\mathrm{Li}, \mathrm{Na}, \mathrm{K})$ distances $(\AA)$ of one $\mathrm{M}$ absorbed on a free benzene molecule.

\begin{tabular}{ccc}
\hline & $\mathrm{E}_{\mathrm{b}}$ & $\mathrm{d}_{\mathrm{M}-\mathrm{C}}$ \\
\hline $\mathrm{Li}$ & 0.89 & 2.33 \\
\hline $\mathrm{Na}$ & 0.89 & 2.82 \\
\hline $\mathrm{K}$ & 0.59 & 3.12 \\
\hline
\end{tabular}

Table S3. Energies of binding (eV) and average M-C $(\mathrm{M}=\mathrm{Li}, \mathrm{Na}, \mathrm{K})$ distances $(\AA)$ of one $\mathrm{M}$ absorbed on a free terephthalic acid molecule.

\begin{tabular}{ccc}
\hline & $\mathrm{E}_{\mathrm{b}}$ & $\mathrm{d}_{\mathrm{M}-\mathrm{C}}$ \\
\hline $\mathrm{Li}$ & -0.43 & 2.27 \\
\hline $\mathrm{Na}$ & -0.18 & 2.65 \\
\hline $\mathrm{K}$ & -0.72 & 2.98 \\
\hline
\end{tabular}


Table S4. The C-C and C-O bond lengths $(\AA)$ on a free terephthalic acid (TA) molecule before and after $\mathrm{M}$ adsorption (M-TA)

\begin{tabular}{llcccc}
\hline & & $\begin{array}{c}d_{\mathrm{C}-\mathrm{C}} \\
\text { (benzene ring) }\end{array}$ & $\begin{array}{c}d_{\mathrm{C}-\mathrm{C}} \\
\text { (benzene-to-carboxy) }\end{array}$ & $d_{\mathrm{C}-\mathrm{O}}$ & $d_{\mathrm{C}-\mathrm{O}(\mathrm{H})}$ \\
\hline Before (TA) & TA & $1.404 / 1.391$ & 1.489 & 1.223 & 1.368 \\
After (M-TA)) & Li-TA & $1.442 / 1.386$ & 1.452 & 1.236 & 1.386 \\
& Na-TA & $1.439 / 1.385$ & 1.448 & 1.238 & 1.390 \\
& K-TA & $1.436 / 1.382$ & 1.447 & 1.240 & 1.393 \\
\hline
\end{tabular}

Table S5. The C-C and C-O bond lengths ( $\AA$ ) of UiO-66 before and after M intercalation (M/UiO-66)

\begin{tabular}{llccc}
\hline & & $d_{\mathrm{C}-\mathrm{C}}($ benzene ring) & $d_{\mathrm{C}-\mathrm{C}}($ benzene to carboxy) & $d_{\mathrm{C}-\mathrm{O}}$ \\
\hline Before & UiO-66 & $1.404 / 1.391$ & 1.496 & 1.278 \\
After & $\mathrm{Li} /$ UiO-66 & $1.432 / 1.390$ & 1.468 & 1.289 \\
& $\mathrm{Na} /$ UiO66 & $1.426 / 1.390$ & 1.472 & 1.288 \\
& $\mathrm{~K} /$ UiO66 & $1.421 / 1.389$ & 1.475 & 1.287 \\
\hline
\end{tabular}

Table S6 The CM5 charges of $\mathrm{M}$ and $\mathrm{C}$ for one $\mathrm{M}(\mathrm{M}=\mathrm{Li}, \mathrm{Na}, \mathrm{K})$ adsorbed on a free benzene molecule

\begin{tabular}{llllllll}
\hline \multicolumn{7}{c}{$\mathrm{M}$} & \multicolumn{7}{c}{$\mathrm{C}$} \\
\hline $\mathrm{Li}$ & 0.316 & -0.135 & -0.136 & -0.181 & -0.135 & -0.136 & -0.180 \\
$\mathrm{Na}$ & 0.182 & -0.139 & -0.124 & -0.125 & -0.140 & -0.124 & -0.123 \\
$\mathrm{~K}$ & 0.252 & -0.131 & -0.148 & -0.131 & -0.131 & -0.149 & -0.132 \\
\hline
\end{tabular}


Table S7. The CM5 charges of $\mathrm{M}, \mathrm{C}$, and $\mathrm{O}$ for one $\mathrm{M}(\mathrm{M}=\mathrm{Li}, \mathrm{Na}, \mathrm{K})$ adsorbed on a terephthalic acid (TA) molecule

\begin{tabular}{llllllllllll}
\hline \multicolumn{3}{c}{ M C of benzene ring } & & & & C & O & O(H) \\
\hline TA & & -0.009 & -0.077 & -0.073 & -0.009 & -0.076 & -0.074 & 0.227 & -0.300 & -0.343 \\
& & & & & & & & & & \\
$\mathrm{Li}$ & 0.604 & -0.086 & -0.128 & -0.126 & -0.086 & -0.128 & -0.126 & 0.184 & -0.340 & -0.368 \\
& & & & & & & & & & \\
$\mathrm{Na}$ & 0.775 & -0.096 & -0.140 & -0.138 & -0.095 & -0.141 & -0.138 & 0.173 & -0.351 & -0.375 \\
$\mathrm{~K}$ & 0.840 & -0.101 & -0.145 & -0.144 & -0.103 & -0.146 & -0.144 & 0.170 & -0.355 & -0.377 \\
& & & & & & & & & & \\
\hline
\end{tabular}

Table S8. The CM5 charges of $\mathrm{Li}, \mathrm{O}, \mathrm{C}$, and $\mathrm{Zr}$ in $\mathrm{UiO}-66-\mathrm{Li}^{\mathrm{a}}(0.5 \mathrm{Li} \text { per node })^{a}$

\begin{tabular}{lccccc} 
& $\mathbf{L i 1}$ & $\mathbf{L i 1}$ & $\mathbf{L i 2}$ & $\mathbf{L i 2}$ & $\mathbf{L i 3}$ \\
\hline $\mathbf{L i}$ & 0.60 & 0.60 & 0.58 & 0.59 & 0.53 \\
\hline $\mathbf{O 1}$ & -0.37 & -0.37 & -0.47 & -0.48 & -0.37 \\
$\mathbf{O 2}$ & -0.79 & - & -0.79 & - & -0.90 \\
$\mathbf{O 3}$ & - & -0.70 & - & -0.70 & - \\
\hline $\mathbf{C 1}$ & -0.07 & -0.07 & -0.03 & -0.03 & -0.02 \\
\hline $\mathbf{Z r}$ & 0.21 & 0.21 & 0.21 & 0.22 & 0.20 \\
\hline & 1.62 & 1.62 & 1.63 & 1.63 & 1.63 \\
\hline & 1.62 & 1.62 & 1.63 & 1.63 & 1.63
\end{tabular}

${ }^{a}$ As with $\mathrm{K}$ intercalation (see Figure 3), the structures of UiO66 with Li intercalations at different positions are denoted $\mathrm{Li} 1 / \mathrm{Li}^{\prime}, \mathrm{Li} 2 / \mathrm{Li} 2^{\prime}$, and $\mathrm{Li} 3$. Only the charges of the atoms near the intercalated $\mathrm{Li}$ are listed. The $\mathrm{O} 1, \mathrm{O} 2$, and $\mathrm{O} 3$ ions are the oxygen atoms closest to $\mathrm{Li}$ in the carboxyl group, the $\mu_{3}-\mathrm{O}$ group, and in the $\mu_{3}-\mathrm{OH}$ group, respectively. The $\mathrm{C} 1$ is the one in benzene nearest to $\mathrm{Li}$, and the $\mathrm{C} 2$ atom is the one in the carboxyl group nearest to $\mathrm{Li}$. 
Table S9. The CM5 charges of $\mathrm{Na}, \mathrm{O}, \mathrm{C}$, and $\mathrm{Zr}$ in $\mathrm{UiO}-66-\mathrm{Na}^{\mathrm{a}}$ (0.5 $\mathrm{Na}$ per node)

\begin{tabular}{llllll}
\hline & $\mathbf{N a 1}$ & $\mathbf{N a 1}$ & $\mathbf{N a 2}$ & $\mathbf{N a 2}$ & $\mathbf{N a 3}$ \\
\hline $\mathbf{N a}$ & 0.76 & 0.77 & 0.73 & 0.73 & 0.84 \\
\hline $\mathbf{O 1}$ & -0.37 & -0.37 & -0.44 & -0.45 & -0.39 \\
$\mathbf{O 2}$ & -0.79 & - & -0.79 & - & -0.87 \\
\hline $\mathbf{C 1}$ & - & -0.70 & - & -0.70 & - \\
\hline $\mathbf{C 2}$ & -0.07 & -0.07 & -0.04 & -0.03 & -0.03 \\
\hline $\mathbf{Z r}$ & 0.21 & 0.21 & 0.20 & 0.21 & 0.18 \\
& 1.62 & 1.62 & 1.62 & 1.61 & 1.61 \\
\hline
\end{tabular}

aAs with $\mathrm{K}$ intercalation (see Figure 3), $\mathrm{Na} 1 / \mathrm{Na} 1{ }^{\prime}, \mathrm{Na} 2 / \mathrm{Na} 2$ ', and $\mathrm{Na} 3$ are the UiO-66 structures with one $\mathrm{Na}$ intercalated at different positions. Only the charges of the atoms near the intercalated $\mathrm{Na}$ are listed. The $\mathrm{O} 1, \mathrm{O} 2$ and $\mathrm{O} 3$ ions are the oxygen atoms closest to $\mathrm{Na}$ in the carboxyl group, in the $\mu_{3}-\mathrm{O}$ group, and in the $\mu_{3}-\mathrm{OH}$ group, respectively. The $\mathrm{C} 1$ and $\mathrm{C} 2$ atoms are respectively the one in benzene nearest to $\mathrm{Na}$ and the one in a carboxyl group nearest to $\mathrm{Na}$. 

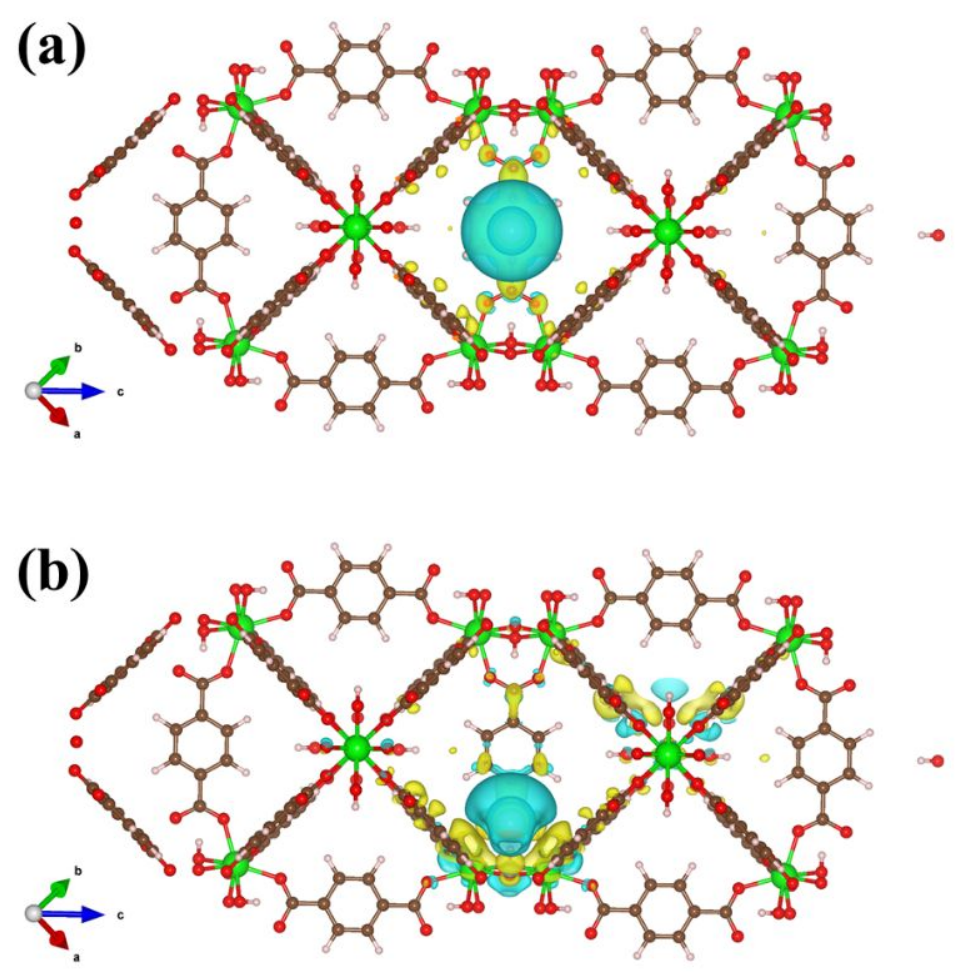

Figure S1. The difference charge density of one K intercalated into the (a) K1 and (b) K3 sites in the $1 \times 1 \times 2$ supercell of UiO-66. The blue and yellow isosurfaces represent electron loss and gain respectively. The isosurface level was chosen to be $0.0008 \mathrm{e} / \AA^{3}$. 

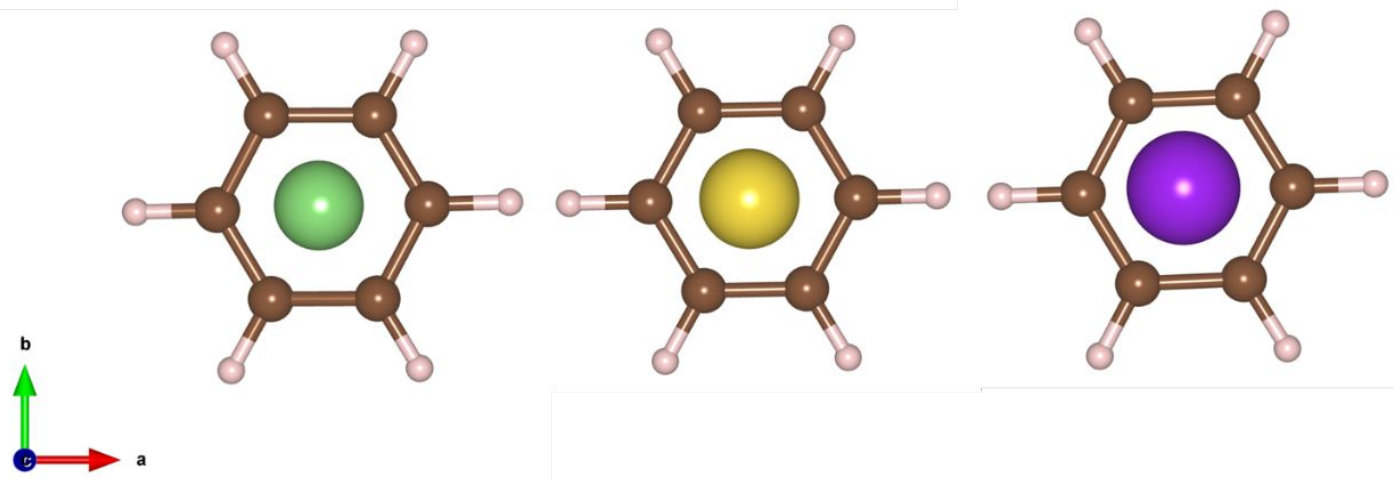

Figure $\mathrm{S} 2$. The configurations of one $\mathrm{Li}, \mathrm{Na}$, and $\mathrm{K}$ adsorbed on a benzene molecule. The green, yellow, purple, brown and white balls represent $\mathrm{Li}, \mathrm{Na}, \mathrm{K}, \mathrm{C}$, and $\mathrm{H}$, respectively.
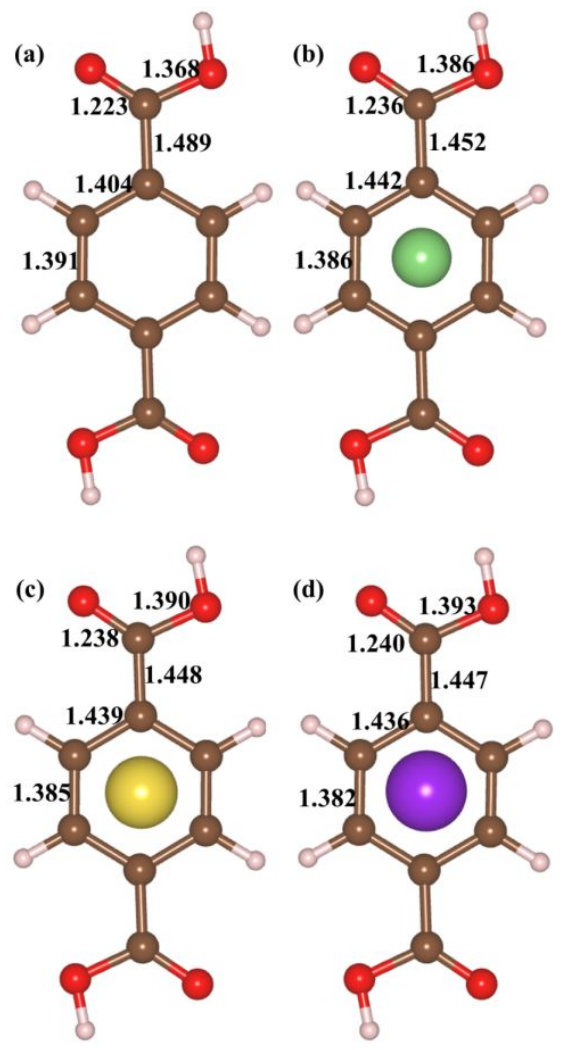

Figure S3. Schematic diagram of (a) free terephthalic acid, and a single (b) Li, (c) Na, or (d) $\mathrm{K}$ adsorbed on the free terephthalic acid molecule. The bond lengths are indicated in Å. The green, yellow, purple, brown, red, and white balls represent $\mathrm{Li}, \mathrm{Na}, \mathrm{K}, \mathrm{C}, \mathrm{O}$, and $\mathrm{H}$, respectively. 

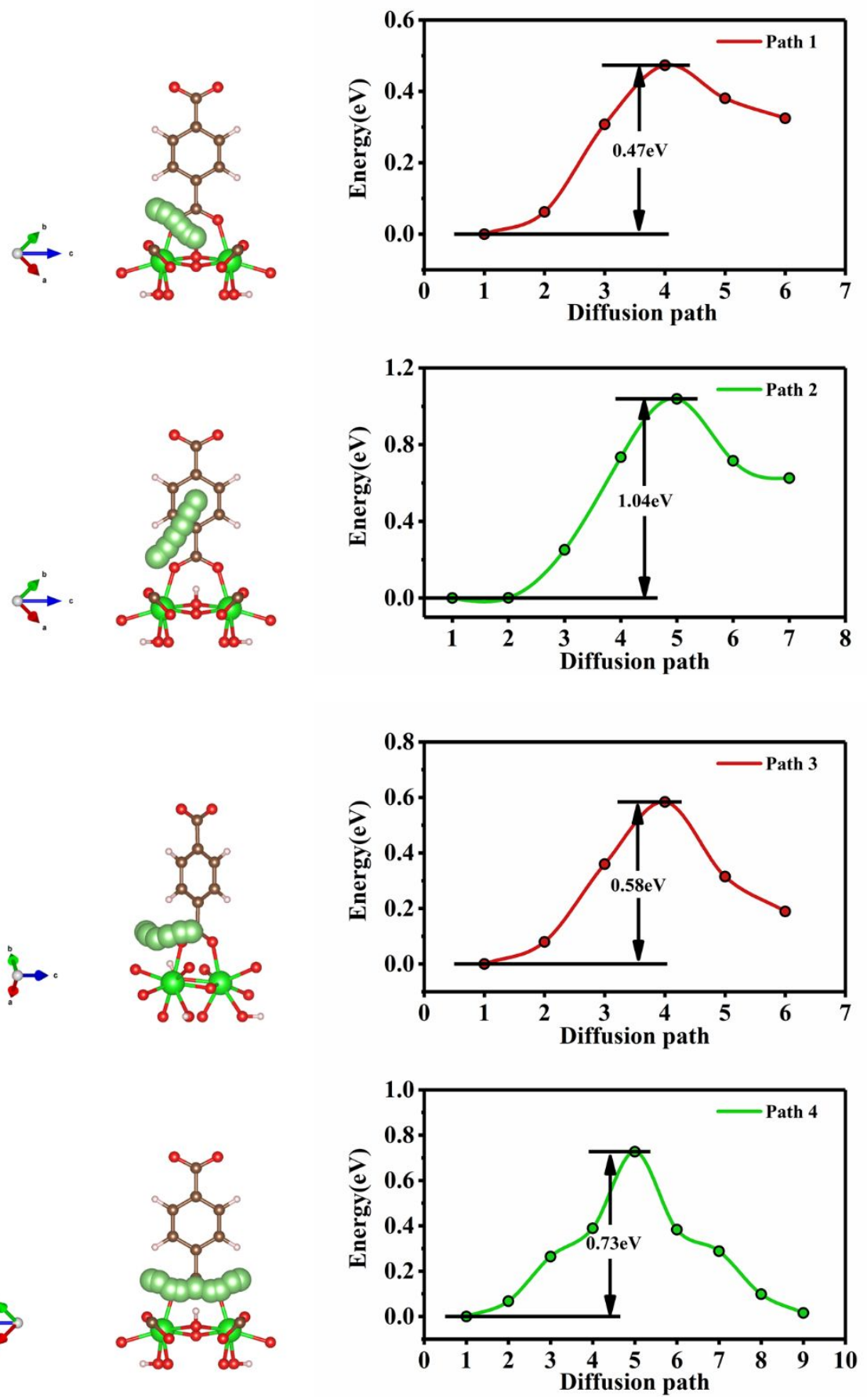

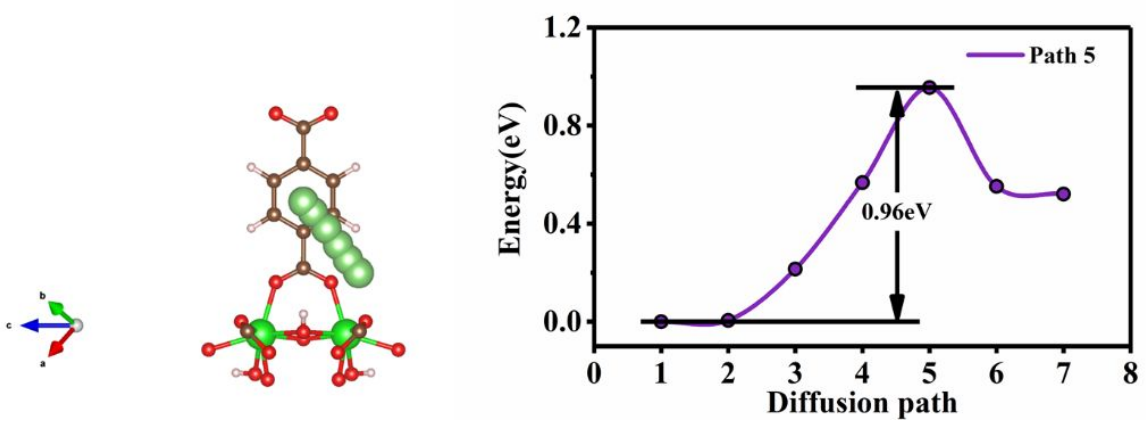

Figure S4. The diffusion paths of one $\mathrm{Li}$ in the $1 \times 1 \times 2$ supercell of UiO-66 and the corresponding energy barriers. 

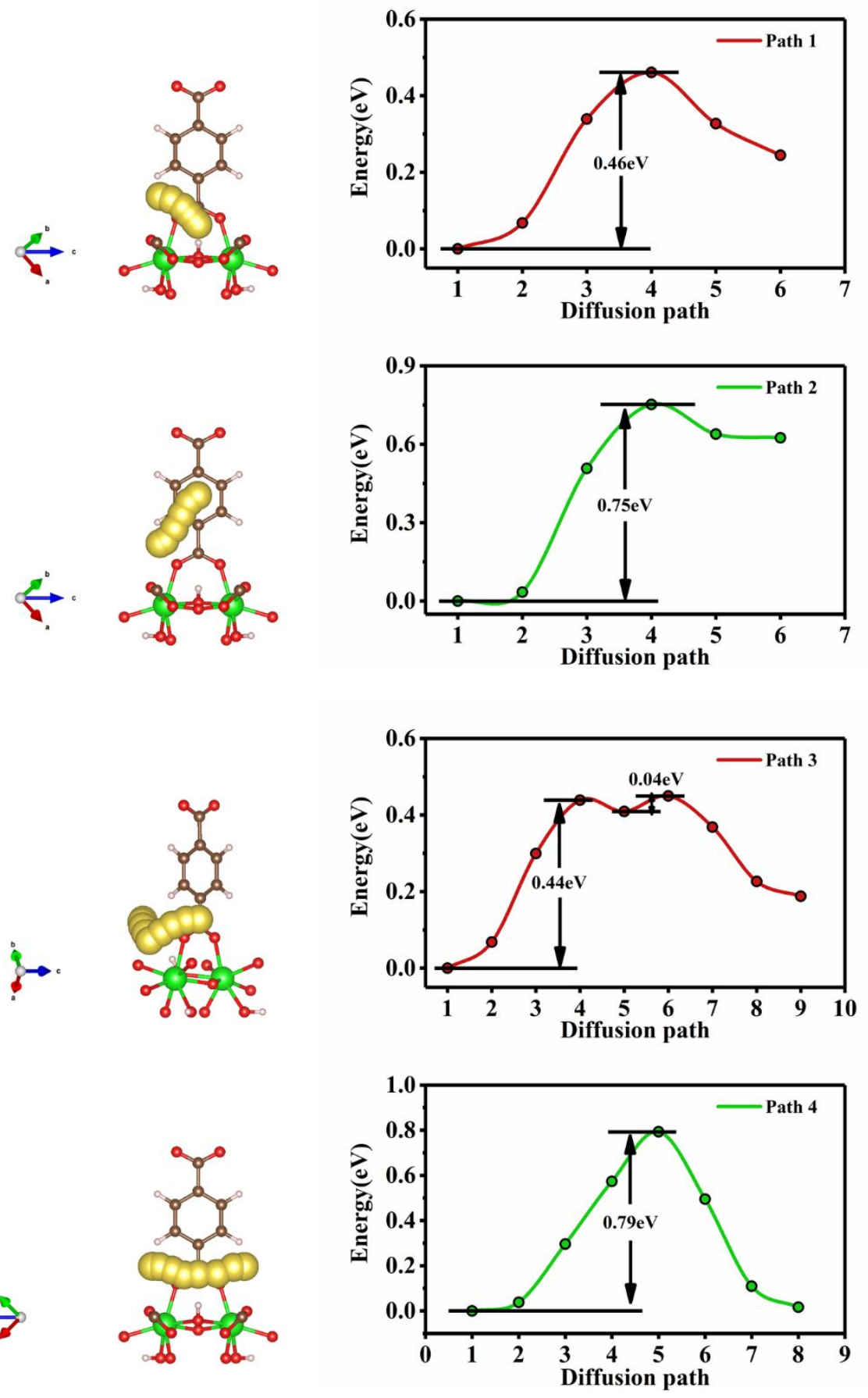

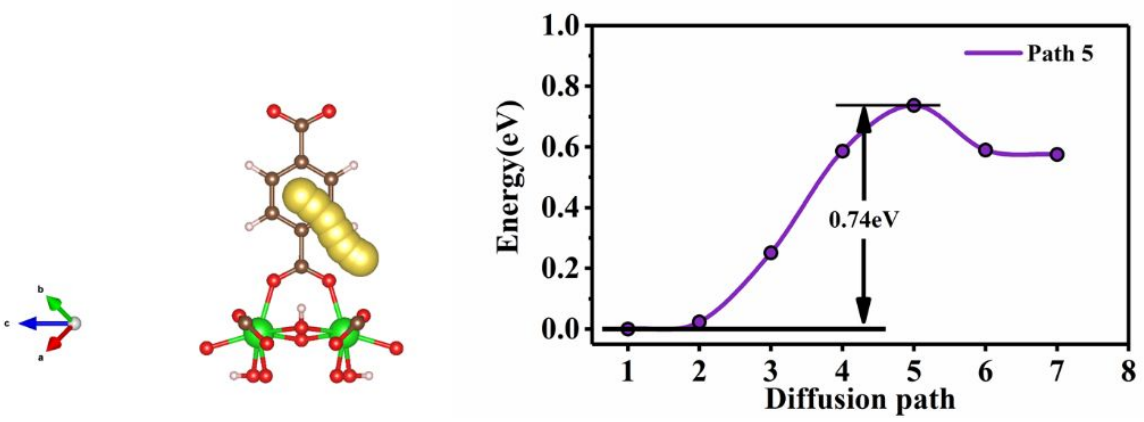

Figure S5. The diffusion paths of one $\mathrm{Na}$ in the $1 \times 1 \times 2$ supercell of UiO-66 and the corresponding energy barriers. 
The structure information (CONTCAR) of $1 \times 1 \times 2 \mathrm{UiO}-66$ primitive cell. UiO-66

1.00000000000000

\begin{tabular}{|c|c|c|c|}
\hline 14.777709 & 870545321 & -0.0054846424097679 & 0.0031800639662801 \\
\hline 7.395078 & 698985342 & 12.7941476459317141 & 0.0029897398446097 \\
\hline 14.781660 & 801778689 & 8.5259337852966048 & 24.1161329383262917 \\
\hline $\mathrm{C}$ & $\mathrm{Zr}$ & & \\
\hline 96 & 64 & & \\
\hline
\end{tabular}

Direct
0.1519919372317557
0.5786552114046134
0.2040606210235279
0.5783643318926535
0.1524356993377516
0.4303718485710647
0.4086393038570305
0.8610070841730093
0.0758037487275868
0.8613788011642235
0.4080118798556605
0.2893650538835189
0.4080913513661071
0.1521016629289562
0.2892713759177084
0.8612290009176847
0.5790780690211514
0.0756884328620144
0.1522697508874288
0.4079030377637802
0.4304837917481592
0.5786786632355526
0.8612222735369788
0.2041571750409594
0.5792830984965761
0.4088013581996031
0.0756378482126944
0.1520002520435113
0.8612906008059487
0.2892824173572253
0.8611939775340095
0.1519139519489519
0.2040856800868148
0.4085421207124420
0.5789895169468652
0.4305078757467010
0.5786619073941771
0.1519805379455424
0.2040674117078303
0.1524384765853705
0.5783724406897919
0.4303651858738032
0.8610103459904968
0.4086537277186060
0.0758044429894364
0.4080055495323931
0.8613872959352877
0.2893591530134594
0.1521004512279348
0.4080929896436734
0.2892691508961180
0.5790614104433038
0.8612323802066904
0.0756897982892079
0.4078834809313581
0.1522746702939617
0.4304979712944520
0.8612194573035351
0.5786749106476495
0.2041580713430291
0.4088079785911848
0.5792818231907743
0.0756384104011248
0.8612810682778923
0.1519998865356363
0.2892852349541153
0.1519125001099672
0.8612014488656934
0.2040843248765883
0.5790186199532229
0.4085430401446034
0.4304985451217330
0.9055874603830625
0.9055864804132884
0.1413630455511518
0.9060694564611897
0.9060481149813264
0.4530539954127815
0.2823534395936261
0.9055971249463300
0.4531181344064674
0.9056194679809072
0.2823421073957821
0.4531136204402983 


\begin{tabular}{|c|c|c|}
\hline & 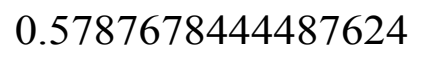 & \\
\hline 89872266300324 & 1518806827319543 & 020150271642 \\
\hline 246 & 8 & 3 \\
\hline 8614453619973954 & & 670 \\
\hline 5 & 1 & 79 \\
\hline 19 & 748 & 329 \\
\hline 402359932029 & 3195301 & \\
\hline & & \\
\hline 5780937941409219 & 461 & \\
\hline & & \\
\hline 224 & 76 & \\
\hline 49465 & 97 & 682 \\
\hline & & 38 \\
\hline & & 391 \\
\hline 3162961 & 414 & 0.5757 \\
\hline & 80 & 086 \\
\hline 1520972626718302 & 889 & \\
\hline 2 & 0.8 & 52 \\
\hline 7270773 & 33 & 85 \\
\hline & 660 & 566 \\
\hline 593 & 0.5 & \\
\hline 8615352341782420 & 280 & (1) \\
\hline 52 & 14 & \\
\hline 669 & 57 & 19 \\
\hline 775 & 0.9 & 11 \\
\hline 21 & 0.9 & 315 \\
\hline & 0.9056 & \\
\hline 0 & 0. & 50 \\
\hline 2319 & 03 & 0.2 \\
\hline & 0.0892163271 & \\
\hline 969 & 0.9 & \\
\hline 9245472835388933 & 0.4461329753742565 & 0.2702436844764984 \\
\hline 0.4461603727959859 & 0.0890032418960921 & 0.2701925424356726 \\
\hline 05 & $0 .$. & 0 . \\
\hline 0.0890896567346910 & 0.4461460700509354 & 0.4620156483916761 \\
\hline 0.5404595373264147 & 0.9244212703177999 & 0.2231871966350358 \\
\hline 0.5408857850540977 & 0.4467476847291508 & 0.0441920784262904 \\
\hline
\end{tabular}




\begin{tabular}{|c|c|c|}
\hline 0 & & \\
\hline 244259679339668 & .0889025112997010 & 829 \\
\hline 6879038060 & 69879934 & 524 \\
\hline 195 & 200259 & 406897551345 \\
\hline 557 & 720 & \\
\hline 242104201453001 & 1373436 & 37209 \\
\hline 82 & 2 & 89 \\
\hline 1 & & \\
\hline & & 30 \\
\hline 38 & & 16 \\
\hline 67 & & \\
\hline & 22 & \\
\hline & & \\
\hline & & \\
\hline 7439 & 72 & 75 \\
\hline 000967348316620 & 0.0040199716333782 & \\
\hline 2359 & 30 & \\
\hline & & \\
\hline 138 & 0004177251 & \\
\hline 68 & 50 & 54 \\
\hline 1384 & 0.00 & \\
\hline & 96 & \\
\hline 241 & 0.6 & 405 \\
\hline 0034907867222406 & 0.0034906693207679 & 964942 \\
\hline & & \\
\hline 3093 & 120 & 180 \\
\hline 0039045073562731 & .0038934099978334 & 0.3460374380095922 \\
\hline 59 & 0.5 & 37 \\
\hline 82 & 0.0 & 321 \\
\hline 16 & 0.0 & 84 \\
\hline 282 & 565 & 133 \\
\hline 3988962704700730 & & 0.2942430691816062 \\
\hline 0.0062476726371894 & 0.5888005981284754 & 0.002 \\
\hline 59508775056077 & 0503710 & 934917 \\
\hline 0.5884616672712417 & 0.0062492376769313 & 0.1995659445784693 \\
\hline 0985154452 & 0.399357780482573 & 0.00298907038623 \\
\hline 0.0062346679465079 & 0.0062381536320507 & 0.2942498155098597 \\
\hline
\end{tabular}




\begin{tabular}{|c|c|c|}
\hline 985 & 264 & \\
\hline 993665122861183 & 5885938750809422 & 0.0029857567712592 \\
\hline 3 & 1 & 1 \\
\hline 408536796129561 & 3006 & \\
\hline 89 & 814 & 25 \\
\hline 87 & 835 & 13 \\
\hline & 243 & \\
\hline & & \\
\hline 0885391690980714 & 7545179 & \\
\hline & 5674 & 616 \\
\hline 400077927662230 & .4458515715110439 & \\
\hline 164 & 302 & 26 \\
\hline & & \\
\hline & 0.54 & \\
\hline 461 & 58951 & 990934 \\
\hline & & \\
\hline & 0.4464291548258414 & \\
\hline 13 & 77 & 00 \\
\hline 0890075626452755 & 257 & 25 \\
\hline & 18 & 398 \\
\hline 260 & 89 & 0. \\
\hline 9244175418761372 & 0.5404322706200806 & .7231904296347583 \\
\hline & 0.5 & \\
\hline 504 & 775 & \\
\hline 986 & 0.92 & 418 \\
\hline 970 & 89 & 749 \\
\hline 42612788 & 0.0042347267700723 & 027 \\
\hline 2 & 729 & 60 \\
\hline 083 & 4899 & 521 \\
\hline & 0.0041949192080926 & \\
\hline 05 & 96 & 9644 \\
\hline 6917009504918282 & 0.0041477935787486 & 0.6500042593045432 \\
\hline 8997887661987 & 0.0045682035838951 & \\
\hline 5743677 & 0.6917555545790701 & 53429254 \\
\hline 0.0034900913932709 & 0.0034997171861953 & 0.6503362346854170 \\
\hline 0.2998730192202039 & 0.6914861075959973 & 0.5023090930228205 \\
\hline 0.6915167956374692 & 0.2998826372475361 & 0.502295861226256 \\
\hline
\end{tabular}




\begin{tabular}{|c|c|c|}
\hline 20 & & \\
\hline 61003800 & .5884892586186363 & 596 \\
\hline & & 566 \\
\hline 923 & 351082 & 3059262827 \\
\hline & & \\
\hline 988775035827192 & 42137 & 817 \\
\hline 67 & 97 & \\
\hline & 449 & \\
\hline & & \\
\hline 37 & 8 & \\
\hline & 10 & \\
\hline & 78 & \\
\hline 25 & & \\
\hline & & \\
\hline 722 & 256 & 504 \\
\hline 13487525479316 & 0.92621 & \\
\hline & 431 & \\
\hline & & \\
\hline 9551532 & 538 & 054 \\
\hline 80 & 27 & 39 \\
\hline 964 & 0.9 & \\
\hline & 550 & \\
\hline 176 & 65 & 395 \\
\hline 9249987827475373 & 0.0787972423599 & 56 \\
\hline & & \\
\hline 323044068211318 & 103 & 0.13 \\
\hline 0796648381689928 & 0.7329714082506784 & 0.4628865893512847 \\
\hline 95 & & 0 . \\
\hline & 0.9 & 240 \\
\hline 40 & 0.2 & 507 \\
\hline 093 & 13 & 5362 \\
\hline 0.2619422330512107 & 0.0794739012559653 & 0.4627510928189760 \\
\hline 0.9258063215078494 & 0.73272600839 & 0.130 \\
\hline 57574069923 & 0.73270727 & 10062958004 \\
\hline 0.9254419906209154 & 0.0792293160698486 & 0.3666243876916015 \\
\hline 0 & 100615120205 & 0.13133161151208 \\
\hline 0.7324694560078654 & 0.2615885828753327 & 0.463173910161809 \\
\hline
\end{tabular}




\begin{tabular}{|c|c|c|}
\hline & 569 & \\
\hline 9 & 202043200006 & 4664780007226083 \\
\hline 44 & 2712 & 6 \\
\hline 327515455255726 & .9327349348426599 & \\
\hline 732 & 067 & \\
\hline 30 & 878 & \\
\hline & & \\
\hline & & \\
\hline 0796854559370288 & 306 & \\
\hline 971 & 423 & \\
\hline 8480 & 93 & \\
\hline 593 & 71690 & 39 \\
\hline & & \\
\hline 03 & & \\
\hline 91 & 71 & 51 \\
\hline & & \\
\hline & ( 261700026510 & -4007 - \\
\hline 57 & 0 & 98 \\
\hline $92500233499^{\prime}$ & 0.07879037 & 76 \\
\hline & 567 & 48 \\
\hline 31 & 0.07 & 0.6 \\
\hline 0797029707458243 & 122 & .962832121 \\
\hline & & \\
\hline 39 & 0.5 & 52 \\
\hline 125 & 0.2 & 08 \\
\hline 215 & 65 & 366 \\
\hline 0.2620241709139653 & 0.0793375070502491 & \\
\hline 0 & 0. & 24 \\
\hline 42381 & 0.732419 & 0.540 \\
\hline 9254395752602415 & & \\
\hline & 0. & 477 \\
\hline 7324907929004152 & 0.2615190487128045 & 0.9631181047229608 \\
\hline 0.2020623395850420 & 0.9323883069507071 & 0.9664703550104761 \\
\hline 75305539 & 0.2020632089799475 & 0.9664706 \\
\hline 0.9323570954776917 & 0.9323664529033149 & 0.6011423613427284 \\
\hline 0.9328014495783327 & 0.9327951012200575 & 0.9664563691748141 \\
\hline .8502246306283205 & 0.0498548639166930 & 0.5250862717571927 \\
\hline
\end{tabular}




$\begin{array}{llll}0.0498719365110759 & 0.8502190154089118 & 0.5250919862777699 \\ 0.0497757929076404 & 0.0497927858602813 & 0.9257730743641513 \\ 0.0498112911784742 & 0.0498048615444394 & 0.5251283483557222 \\ 0.1193604423743149 & 0.8804648328702156 & 0.0598236837262905 \\ 0.8804888384022840 & 0.1194825068711573 & 0.4404173964104657 \\ 0.1194363286943156 & 0.1194213110174417 & 0.4404350917331941 \\ 0.8805410119851323 & 0.8805354365126248 & 0.0597750527251397 \\ 0.8804667804506580 & 0.1193604136571652 & 0.0598241689166979 \\ 0.1194868306848070 & 0.8804852720715410 & 0.4404233659047418 \\ 0.1192687889320709 & 0.8804649784671413 & 0.5598544924925093 \\ 0.8805865343803043 & 0.1195108402168795 & 0.9403761086772690 \\ 0.1194680105231990 & 0.1194715745350171 & 0.9404488039529005 \\ 0.8804697040425842 & 0.8804646955856352 & 0.5597899422690592 \\ 0.8804650592289089 & 0.1192605518433945 & 0.5598495746382652 \\ 0.1195089444418056 & 0.8805966416604237 & 0.9403742660413378\end{array}$


The structure information of $1 \times 1 \times 2 \mathrm{UiO}-66$ primitive cell with one $\mathrm{K}$ at $\mathrm{K} 1$ site. UiO-66-K

1.00000000000000

$\begin{array}{lll}14.7789027980964711 & -0.0010274300018388 & 0.0003265843753374\end{array}$

$\begin{array}{lll}7.3995321190031191 & 12.7902795669356468 & 0.0004604110102840\end{array}$

$14.7807674072022497 \quad 8.5242488580871267 \quad 24.1198978320153046$

$\mathrm{H} \quad \mathrm{C} \quad \mathrm{O} \quad \mathrm{Zr} \quad \mathrm{K}$

$\begin{array}{lllll}56 & 96 & 64 & 12 & 1\end{array}$

Direct
0.1521101275806184
0.5784418278288541
0.2040292684907851
0.5786537637452474
0.1520820815216429
0.4301741483741792
0.4088374681821106
0.8609641673758880
0.0758562301921853
0.8615642469217254
0.4079771221869977
0.2892493256510498
0.4079783186436850
0.1521871150838026
0.2892568958276041
0.8613476722226304
0.5792594357249953
0.0756878756104875
0.1522470116403279
0.4078206737666648
0.4303802193719509
0.5787249962754060
0.8612451744768371
0.2040895197177585
0.5795546265006442
0.4089806796582408
0.0756663322320403
0.1525185553182994
0.8615422583289549
0.2890590951648253
0.8612314990544908
0.1521428281222156
0.2040391051439616
0.4092748667285774
0.5791410636587729
0.4300633383400173
0.5784694055259815
0.1520682894314734
0.2040742990912889
0.1520986204280219
0.5785811187861626
0.4301963454960661
0.8610130838453737
0.4088498969140502
0.0758370692808869
0.4080735424783710
0.8614788620852679
0.2892315690809565
0.1522272903765143
0.4079654617124820
0.2892265356972051
0.5792293584130137
0.8612750441409823
0.0757025417635425
0.4079441324387814
0.1522003491834513
0.4303606976408988
0.8612921240037406
0.5786658392213838
0.2041057219225426
0.4089579714068009
0.5795746680092884
0.0756600543210138
0.8616247869257655
0.1524419281441281
0.2891040929170392
0.1521214098653088
0.8612616006448724
0.2040013596494056
0.5801988771687401
0.4098721173914801
0.4295608085281495
0.9056016476169179
0.9055642628218975
0.1413711347283837
0.9063836973910496
0.9061113332190968
0.4528567688041511
0.2826331759707011
0.9050745452534698
0.4530829257378056
0.9056409814066235
0.2822601902343088
0.4530311468487414 


\begin{tabular}{|c|c|c|}
\hline 42168 & 20887169 & \\
\hline 791115530649450 & 1516019943911389 & 0.9300551867628527 \\
\hline 359 & 59 & 4 \\
\hline 8617385313714857 & & 875 \\
\hline 6 & 0 & 62 \\
\hline 05 & 569 & 766 \\
\hline 1028 & 43438 & 588 \\
\hline & & \\
\hline & 307 & \\
\hline & & \\
\hline 68 & & \\
\hline 050 & 709 & 527 \\
\hline & & \\
\hline & & \\
\hline & 0478391 & 2557 \\
\hline & 090 & 68 \\
\hline 9389 & 5 & \\
\hline 62 & 87 & 68 \\
\hline 904 & 07 & \\
\hline & 594 & \\
\hline 93 & 0.5779 & 77 \\
\hline 8614707603093088 & .1525262155160 & \\
\hline & & \\
\hline 318 & 72 & \\
\hline 216 & 052 & 359 \\
\hline 825 & 0.9 & 0 . \\
\hline 0.2823070374791124 & 0.9054970 & \\
\hline 59 & 0 . & \\
\hline 38 & 203 & 0.2 \\
\hline & 0.0896053367 & \\
\hline & 0.9 & \\
\hline 9246101893951479 & 0.4461743792570611 & 0.2702050793813427 \\
\hline 0.4461352359041684 & 0.0890104676858252 & 0.2702016027190579 \\
\hline & 44 & 0. \\
\hline 0.0895644273323790 & 0.4456250830401408 & 0.4621654596976639 \\
\hline 0.5405227710160576 & 0.9244058950958244 & 0.22316512285514 \\
\hline 0.5409989623467241 & 0.4468225628717809 & 0.044293939363226 \\
\hline
\end{tabular}




\begin{tabular}{|c|c|c|}
\hline 587 & 691054 & \\
\hline 251078211 & 0891096612710912 & 02230465218052188 \\
\hline 52 & 0 & 0 \\
\hline 403643271140742 & .0889285424929729 & \\
\hline 26 & & 7720 \\
\hline 743 & 874 & 3994 \\
\hline 557 & & 301 \\
\hline 26 & & \\
\hline 5410150166149893 & 0.92430149887 & \\
\hline 1 & 0 & \\
\hline & & \\
\hline 1822 & 941 & 0.04 \\
\hline & & \\
\hline 53493 & & \\
\hline 007389007 & 17257667165 & 5447 \\
\hline & & \\
\hline ח & 408 & \\
\hline 96 & 50 & 10 \\
\hline 5703 & 87 & 30 \\
\hline 600 & 32 & 500 \\
\hline 22 & 0.0 & 98 \\
\hline 236752 & 0.0045129056736063 & 0.3459075768995091 \\
\hline & & \\
\hline 264 & 1 & 48 \\
\hline 081 & 0.6 & 501 \\
\hline 770 & 0.3 & 596 \\
\hline 61164491 & 0.0039705229280915 & \\
\hline 26 & 0.5 & 33 \\
\hline 253 & 0.00 & 985 \\
\hline & & \\
\hline 565 & & 3096 \\
\hline 3989192398449940 & 0.0063942434075010 & 0.2942715791361313 \\
\hline 0.0062002694738321 & 0.5889708807704513 & 0.002723359 \\
\hline 1759 & 0.3996487454328165 & 0.0028502734094312 \\
\hline 0.5885111645669699 & 0.0062448895977909 & 0.1995429485866397 \\
\hline 0.5885702531478874 & 0.3991820150141059 & 0.0031804136772830 \\
\hline 0.0065784893425170 & 0.0064893982827620 & $0.294116381824558 \mathrm{C}$ \\
\hline
\end{tabular}




\begin{tabular}{|c|c|c|}
\hline 59 & & \\
\hline 96 & 607 & 2 \\
\hline 306 & 1442 & 68 \\
\hline 7055 & 036 & 17137202607001 \\
\hline 4 & 1 & 59 \\
\hline 9250153284941898 & .4456016706869703 & 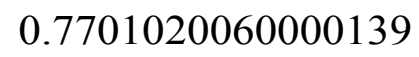 \\
\hline 22 & 98 & 90 \\
\hline 643 & 315 & 75 \\
\hline 67996 & 55606 & 049 \\
\hline & 074 & 967 \\
\hline 4329 & 0.4457273074666767 & \\
\hline 4481 & 791 & 260 \\
\hline & & \\
\hline & & \\
\hline 8 & 4 & 93 \\
\hline & & \\
\hline & 269 & 362 \\
\hline 77 & $0 . S$ & 0.17 \\
\hline 0893108472936170 & 702020754 & 0.770 \\
\hline & 9 & 96 \\
\hline & 0.0 & \\
\hline 151092797414 & 0.53995677442 & 0.72 \\
\hline 339 & 13 & 603 \\
\hline 9248371824924667 & 0.089 & 309 \\
\hline & & \\
\hline 30 & 8 & 0.9 \\
\hline 53 & 0.0 & 5183 \\
\hline & & \\
\hline 039671792701170 & 54770 & 0.650 \\
\hline 917149670293198 & 0.0042520633631979 & 0.5019446790202741 \\
\hline 390 & 3 & 438 \\
\hline 6916136955063036 & 0.0041220009011284 & \\
\hline 001 & 0.004735 & 279 \\
\hline 0.004213736260 & 0.69 & 32637 \\
\hline 0.0035897226020155 & 0.0035835248798067 & \\
\hline & 0.69114896609008 & 0.5025756135661392 \\
\hline 0.6921663345094909 & 0.2997168511159622 & 0.5021422350061167 \\
\hline
\end{tabular}




\begin{tabular}{|c|c|c|}
\hline 54 & 4594 & \\
\hline 064168962005489 & 5881984618587284 & 60042412004 \\
\hline 57 & 22 & 6 \\
\hline 4967874851491 & .0067278724502743 & 454 \\
\hline 2 & 1 & 12 \\
\hline 75 & 565278 & \\
\hline & & \\
\hline & & \\
\hline 5881024626105721 & 0.0065732163530652 & \\
\hline 58 & 095 & \\
\hline 03 & & \\
\hline 6644 & 133 & 70 \\
\hline & & \\
\hline & & \\
\hline 108048318 & 126311 & 67022 \\
\hline & & \\
\hline & ( 26120 & \\
\hline 5 & 68 & 22 \\
\hline 359859 & 0.7327 & \\
\hline & 563 & 040 \\
\hline 67946 & 79 & 0 \\
\hline 7328518706931005 & .262071304 & .0399009732353490 \\
\hline 59 & 1 & \\
\hline 4 & 1 & 80 \\
\hline 275 & 0.73 & 3695 \\
\hline 361 & 03 & 542 \\
\hline 0.0797894006904635 & 0.7327426093142942 & 255393 \\
\hline 4 & 0.2 & 681 \\
\hline 1006 & 0.926 & 0.3 \\
\hline 0.0800423258655713 & 0.2619229746041830 & \\
\hline & 0. & \\
\hline & 0.0796535852936555 & 0.4626438280614698 \\
\hline 8451593505652 & 0.7327150556296013 & \\
\hline 56870406 & 0.7328435258444702 & 0.0398926006774865 \\
\hline 0.9254490618906569 & 0.0792547666222338 & 0.3666066001315239 \\
\hline 0.0788608677740933 & 0.9249552320433734 & 0.1313353407553953 \\
\hline .7333529375611292 & 0.2607581207779365 & 0.462906193399699 \\
\hline
\end{tabular}




\begin{tabular}{|c|c|c|}
\hline 424 & 0 & 0 \\
\hline 957 & 386 & 93 \\
\hline 678 & 31843 & 132 \\
\hline 1188 & 964 & 7618 \\
\hline 1 & 55 & 37 \\
\hline 0498467689241245 & .8504915779813993 & .02506414514 \\
\hline & 577 & 96 \\
\hline 219 & 52 & 42 \\
\hline 96389616205188 & 7323546325632464 & 556 \\
\hline 43 & & 720 \\
\hline & & 259 \\
\hline & 802 & 5363 \\
\hline & & \\
\hline & 33 & \\
\hline 54 & 1 & 96 \\
\hline 16 & $0.92570^{\prime}$ & \\
\hline & 374 & \\
\hline & & \\
\hline 9249517610475755 & 0.07896320 & 12 \\
\hline & 9 & \\
\hline & & \\
\hline 9771079 & 27581 & 9615 \\
\hline & 30 & 38 \\
\hline 817 & 0.9264 & \\
\hline & 0. & 567 \\
\hline 28 & 34 & 03 \\
\hline 620327127 & 3210 & 8651486617 \\
\hline & & \\
\hline 2608586234213395 & 0.732698996 & 384 \\
\hline 0.9254214387323310 & 0.0797289221351761 & 0.866490852 \\
\hline 95 & 5 & 0. \\
\hline 0.7325317039506996 & 0.2615129129946940 & 0.9631776485221316 \\
\hline 744 & 0.9323917738870943 & 075 \\
\hline 323848221058135 & 0.2020071404336247 & 581559008 \\
\hline 0.9323960195448305 & 0.9323753898608483 & \\
\hline & 0.3 & 0.96 \\
\hline 0.8501709792104213 & 0.0498786111590749 & 0.5250388222335736 \\
\hline
\end{tabular}




$\begin{array}{llll}0.0500067928027078 & 0.8500186390358817 & 0.5250666976376963 \\ 0.0497872475927777 & 0.0498125623932607 & 0.9257707687539561 \\ 0.0499401282655808 & 0.0497894833964855 & 0.5250751000829313 \\ 0.1193488595209384 & 0.8803105439533421 & 0.0599777170876546 \\ 0.8806808401450292 & 0.1197014458328055 & 0.4402106386885075 \\ 0.1196304182547583 & 0.1194344117329364 & 0.4402575707972509 \\ 0.8804345378035002 & 0.8804594499187536 & 0.0598734631873202 \\ 0.8803257647859629 & 0.1193489589370396 & 0.0599840108734415 \\ 0.1199813527430099 & 0.8802862904908977 & 0.4402578852796958 \\ 0.1196066141447346 & 0.8803725459248862 & 0.5597183804108332 \\ 0.8804130552491007 & 0.1194530833265202 & 0.9405461869739020 \\ 0.1193011730694806 & 0.1193066283975309 & 0.9407430160984968 \\ 0.8807288742767898 & 0.8806175333669921 & 0.5595858961901969 \\ 0.8805664642817368 & 0.1194889676956708 & 0.5596921798785692 \\ 0.1193952738917596 & 0.8804694721143498 & 0.9405378010522321 \\ 0.3675840427573813 & 0.3574426665449700 & 0.5681486146582907\end{array}$


The structure information of $1 \times 1 \times 2 \mathrm{UiO}-66$ primitive cell with one $\mathrm{K}$ at $\mathrm{K} 1{ }^{\prime}$ site. UiO-66-K

1.00000000000000

$\begin{array}{lll}14.7794395407738666 & -0.0037264613746214 & 0.0007727189318995\end{array}$

$\begin{array}{lll}7.3974652950677866 & 12.7940630297666527 & 0.0006707041503167\end{array}$

$14.7804748841684983 \quad 8.5249480061612068 \quad 24.1193264737743895$

$\mathrm{H} \quad \mathrm{C} \quad \mathrm{O} \quad \mathrm{Zr} \quad \mathrm{K}$

$\begin{array}{lllll}56 & 96 & 64 & 12 & 1\end{array}$

Direct
0.1520075024117881
0.5784898213909043
0.2039233470714377
0.5792164694255847
0.1514487217074361
0.4300989596604880
0.4087102950947284
0.8610465396884028
0.0757926676679747
0.8611497251381957
0.4081821669380838
0.2894062784032344
0.4079568266186670
0.1521155355681436
0.2891929053337231
0.8613312203758285
0.5792463508560479
0.0756123000358689
0.1513421937156531
0.4088020812308391
0.4301556485573698
0.5791369947365118
0.8608401500534504
0.2042073843907764
0.5796958441097673
0.4092440945349926
0.0756258907966017
0.1523669866876084
0.8615863501425997
0.2891098711241071
0.8613707140320388
0.1520712147857840
0.2039732157077777
0.4064981580436445
0.5766917049488023
0.4314164875376673
0.5784970115542558
0.1519939176165350
0.2039331868736937
0.1514466159339923
0.5792231430977302
0.4301090818599540
0.8610800900389456
0.4087983793039361
0.0757739824957270
0.4082836561224116
0.8610549196865509
0.2894313794476129
0.1521581814337678
0.4079215349051007
0.2891708555769221
0.5791528562825230
0.8612320784915347
0.0756599534156000
0.4088233662755216
0.1513195365660683
0.4301382612859841
0.8609140925182449
0.5790563552454139
0.2041851520627800
0.4091833679479606
0.5797310164674435
0.0756226687344761
0.8615762915340777
0.1522493262388822
0.2891481390270443
0.1521682946794430
0.8614015282092375
0.2039379513664712
0.5768084762988858
0.4070303089215120
0.4312648337500152
0.9058464659411765
0.9058217333687626
0.1411482256129413
0.9059859961511360
0.9060184484275214
0.4531611154029467
0.2813167271198853
0.9064966833215952
0.4531740391846293
0.9064504924943789
0.2814037983140821
0.4531647577415213 


\begin{tabular}{|c|c|c|}
\hline 40 & 057 & \\
\hline 722 & 9426 & 0 \\
\hline 081134279402583 & .8614867053988084 & 377 \\
\hline 32 & 31 & 04 \\
\hline 5548 & .1520837001478497 & 5729 \\
\hline 3614478013108543 & 637 & \\
\hline & & \\
\hline 3 & 41 & \\
\hline & & \\
\hline & & \\
\hline & & \\
\hline 73357125094618 & 940259 & 203 \\
\hline 787470622642013 & 0.1517591830070088 & \\
\hline 533 & 763 & 847 \\
\hline & 3 & 56 \\
\hline 4078658867525484 & 0.861538 & 48 \\
\hline 242 & 69 & 95 \\
\hline 0468 & 03 & \\
\hline & & \\
\hline 62 & 0.5 & 70 \\
\hline 6750635521580 & 79143743718230 & 0.5767730269 \\
\hline & & \\
\hline 96 & 35 & 064 \\
\hline 778188480252099 & 28 & 398 \\
\hline & 7 & \\
\hline 12 & 0.9 & 25 \\
\hline 3 & 0.9 & 0 . \\
\hline 663 & 083 & 133 \\
\hline & & \\
\hline 7 & 0. & 0 . \\
\hline .4467753799529504 & $0.924210267^{\prime}$ & 1514 \\
\hline 0.9237457052416275 & 0.4468888245645640 & 0.2705450157100879 \\
\hline 650507 & 0.0885849 & 5753 \\
\hline 0.9244217158755454 & 0.5409840279889940 & 0.0440796202140683 \\
\hline 0.0881427431692898 & 0.4470606502055634 & 0.4616883019569330 \\
\hline 014071150 & 0.9 & \\
\hline 0.5410554838662540 & 0.4469324216718407 & 0.0443025936856192 \\
\hline
\end{tabular}




\begin{tabular}{|c|c|c|}
\hline 00524 & 62741 & \\
\hline 244624010964960 & 0889314280546885 & .2230777958804960 \\
\hline 13 & 04135845794 & 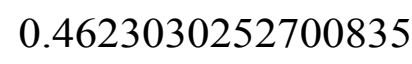 \\
\hline 407071731503135 & 790003255 & \\
\hline 833 & 49 & \\
\hline 689 & 30 & 002 \\
\hline & & \\
\hline & & \\
\hline 5409140883354968 & 0.9243448956738826 & \\
\hline 61052 & 641 & \\
\hline 30255136 & 001 & \\
\hline 74719 & 398 & 0.04 \\
\hline & & 21 \\
\hline 29 & & 0.22 \\
\hline 2747 & 37136 & 0.46 \\
\hline & & \\
\hline 0042521479458969 & 020006720555 & 0.3460 \\
\hline 97 & 0 & 70 \\
\hline 691974323 & 58 & 67 \\
\hline 58 & 47 & \\
\hline 804 & 0.0 & 0.1 \\
\hline 000746642696511 & 0.0042277552797921 & 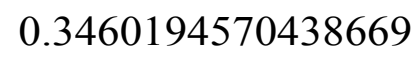 \\
\hline & 0. & \\
\hline 18 & 82 & \\
\hline 4091 & 0.6 & 0.0022 \\
\hline 92 & 0.3 & 0.0 \\
\hline 0039077689453718 & 0.0039512 & \\
\hline 4 & 0.5 & 0 . \\
\hline 85 & 0.00 & 0.00 \\
\hline & & \\
\hline & & \\
\hline 3994280204073064 & 0.0057282801534010 & 0.2945740065767487 \\
\hline 0.0062435804745268 & 0.588974204 & 0.002685 \\
\hline 2555 & 0.3995630 & 0.002832687 \\
\hline 0.5890775094378878 & 0.0056416806937726 & 0.1997166882938493 \\
\hline 0.5885227796301591 & 0.3991593653268125 & 0.00325236591204 \\
\hline 0.0063558736385639 & 0.0063150904208148 & 0.294227212445502 \\
\hline
\end{tabular}




\begin{tabular}{|c|c|c|}
\hline 021 & 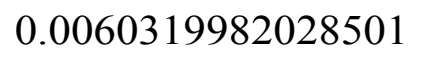 & \\
\hline 991457432964777 & 5885328559507490 & 597068 \\
\hline 51 & 2 & 3 \\
\hline 410209970601708 & .0885329491332632 & 9617265669340551 \\
\hline 310 & 76 & \\
\hline 69 & 635 & 322 \\
\hline & & \\
\hline & & \\
\hline 0884135314977709 & 793 & \\
\hline 908 & 777 & 574 \\
\hline 2390 & 85 & \\
\hline 160 & 649 & 369 \\
\hline & & 34 \\
\hline 682 & 0.53 & 043 \\
\hline & 416 & 0.72317 \\
\hline & & \\
\hline & 5640334 & 051 \\
\hline 56 & 0.9 & 27 \\
\hline 08899228 & 0.446 & 04 \\
\hline & 74 & \\
\hline 15 & 0.0 & 0. \\
\hline 9244287702317087 & 63 & .7232107987691759 \\
\hline & & \\
\hline 40 & 50 & 08 \\
\hline 93 & 0.924 & 0 . \\
\hline 242 & 0.4 & 341 \\
\hline 01069572934168 & 0.0040364716479623 & \\
\hline 73 & 0.2 & 0 . \\
\hline 76 & 0.6 & 607 \\
\hline & 0.0039292907207846 & \\
\hline 07 & 0.3 & 179 \\
\hline 6914919076163346 & 0.0040833445678452 & 0.6500173564409979 \\
\hline 0.2999116618680906 & 0.0046098580288271 & 0.8459 \\
\hline 5031 & 586755042273 & 0.5018626776009285 \\
\hline 0.0035358482138919 & 0.0035266571601827 & 0.6503327032042290 \\
\hline 0.3002655410775523 & 0.6925607377876313 & 0.5019624409130152 \\
\hline 0.6926282183630720 & 0.3007803552002872 & 0.5018133825806449 \\
\hline
\end{tabular}




\begin{tabular}{|c|c|c|}
\hline 24 & & \\
\hline 6156073038 & .5884650501112914 & 378 \\
\hline 892921211064722 & .0057714394442456 & 7381 \\
\hline 50731 & 302 & 3831623096 \\
\hline 679 & 13 & \\
\hline 3987815047530100 & & 474 \\
\hline & 8 & 29 \\
\hline & 686 & \\
\hline & & 14 \\
\hline 05 & & \\
\hline 92 & & \\
\hline & & \\
\hline 7125 & 23 & \\
\hline & & \\
\hline 12 & 342 & $0.402 c$ \\
\hline 12600250808754 & 0.9263609948 & \\
\hline & 192 & \\
\hline & & \\
\hline 3333656 & 0722712100 & \\
\hline 38 & 77 & 64 \\
\hline 2480 & 479 & 074 \\
\hline & 677 & \\
\hline 386 & 65 & 889 \\
\hline 40 & 3 & 39 \\
\hline & & \\
\hline 7323256994270664 & 303 & 0.13 \\
\hline 0794128829372149 & 100658 & 0.4627599602545314 \\
\hline 368 & 45 & \\
\hline 511 & 0.9 & 234 \\
\hline 84 & 0.2 & 322 \\
\hline 726 & 536 & 832 \\
\hline 0.2619396456792873 & 0.0794451393863782 & 0.4626580677451768 \\
\hline 0.92555057 & 0.7327384622 & 0.13096 \\
\hline 372675865897 & 0.7329135915145248 & 0.0399262043232252 \\
\hline 0.9252692912234038 & 0.0792591739889606 & \\
\hline 1 & 02700 & 0.13131972219803 \\
\hline 0.7338536263023384 & 0.2618568725925363 & 0.4625238735827735 \\
\hline
\end{tabular}




\begin{tabular}{|c|c|c|}
\hline 10 & & \\
\hline 31500607 & 753 & 671 \\
\hline 870 & .9324691227297439 & $0.101007 \epsilon$ \\
\hline 4 & 774 & 32 \\
\hline 466 & 353 & 64201 \\
\hline 09589 & & 717 \\
\hline & & \\
\hline 5 & 863 & \\
\hline & & \\
\hline 33 & 98 & 27 \\
\hline 162 & 14 & 525 \\
\hline 856 & 465 & 05 \\
\hline 619194208244096 & 0.0799905542 & \\
\hline 4633 & 709 & 0.539 \\
\hline & & \\
\hline 3972 & 0.9256 & 07 \\
\hline 776 & 104 & 979 \\
\hline & 24 & \\
\hline & ( 0700 & \\
\hline 1197 & 0.73 & 88 \\
\hline 7323088081611513 & 0.0798435620393694 & 002 \\
\hline & 10 & \\
\hline 81760 & 99 & 65 \\
\hline 2614452669099153 & 47058 & \\
\hline & 13 & \\
\hline 19709 & 0.9 & \\
\hline 55 & 0. & 0 . \\
\hline 321 & 0.73 & 0.63 \\
\hline & 0.7345091919804634 & \\
\hline 7 & 0. & 0 \\
\hline 788811805884701 & 0.924996 & 0.6313080 \\
\hline 0.7324949381203896 & 0.2616112620743810 & \\
\hline 0364167977177 & 0.932447 & 574491 \\
\hline 0.9324124928514762 & 0.2020873921919081 & 0.9664658291404707 \\
\hline 0.9322652474936647 & 0.9323193554357375 & 0.6012010318108463 \\
\hline 0 & 0.9 & 0.9 \\
\hline 0.8501944480913224 & 0.0498712442123382 & 0.5251019842902026 \\
\hline
\end{tabular}




$\begin{array}{llll}0.0498057165149653 & 0.8502304940101615 & 0.5250977682648974 \\ 0.0497606710882602 & 0.0498239468686510 & 0.9257708269455321 \\ 0.0496902026413123 & 0.0498253004502767 & 0.5252615438734127 \\ 0.1194843346787323 & 0.8806111536682294 & 0.0596691362902662 \\ 0.8801614822808174 & 0.1194378497626426 & 0.4405219885350196 \\ 0.1191435725577507 & 0.1192178524547180 & 0.4405768629117146 \\ 0.8806739506011365 & 0.8806772017148777 & 0.0595330291294280 \\ 0.8806220533034089 & 0.1194909813864360 & 0.0596708623088572 \\ 0.1193074350229809 & 0.8802499621287714 & 0.4405151066886787 \\ 0.1191511659407444 & 0.8802254306066871 & 0.5600343377830780 \\ 0.8805815009322698 & 0.1196906286216630 & 0.9402736573964554 \\ 0.1195761087450584 & 0.1196042372856944 & 0.9403348098031442 \\ 0.8803451536745639 & 0.8803711697853847 & 0.5599390145798417 \\ 0.8801102585019507 & 0.1192703606413561 & 0.5600349987485205 \\ 0.1196366952306544 & 0.8806661353328241 & 0.9402545503447629 \\ 0.6215081620372407 & 0.6249419679088550 & 0.4381582526872722\end{array}$


The structure information of $1 \times 1 \times 2 \mathrm{UiO}-66$ primitive cell with one $\mathrm{K}$ at $\mathrm{K} 2$ site. UiO-66-K

1.00000000000000

\begin{tabular}{|c|c|c|c|c|c|}
\hline \multicolumn{4}{|c|}{14.7825847353168172} & -0.0091793428715875 & 0.0083307642694916 \\
\hline \multicolumn{4}{|c|}{7.3943205583113496} & 12.7962320021332481 & 0.0058127634290669 \\
\hline \multicolumn{4}{|c|}{14.7928060848563145} & 8.5253215358400762 & 24.1139609180108145 \\
\hline & $\mathrm{C}$ & $\mathrm{O}$ & $\mathrm{Zr}$ & K & \\
\hline & 96 & 64 & 12 & 1 & \\
\hline
\end{tabular}

Direct
0.1506404205172638
0.5783856128330455
0.2039259017493032
0.5955764710053633
0.1308647057246884
0.4232783474997548
0.4082978717357516
0.8611470305492048
0.0757314654094225
0.8603309747518189
0.4070009980317001
0.2899309962438960
0.4080627086553032
0.1524114473997439
0.2890226833822094
0.8609156724526630
0.5793170336529034
0.0755975615720744
0.1512930978942647
0.4103854730385575
0.4290363000297082
0.5781733983583670
0.8613985576342266
0.2041116189939065
0.5806646454849919
0.4109194451315682
0.0749857815390560
0.1508734091414881
0.8616272809387422
0.2895052493300379
0.8615458696771378
0.1530628918163008
0.2036883476424202
0.4217363791168448
0.5920495572031703
0.4248215351396510
0.5786067208309376
0.1521253838652319
0.2037592162539661
0.1512119945841353
0.5805675901585103
0.4293319982798347
0.8607910925660004
0.4087716819623129
0.0757995676392719
0.4074025185070989
0.8618013587134692
0.2893126277707828
0.1510996280226370
0.4080636393493715
0.2892716762491174
0.5785962090954999
0.8613714498713808
0.0756572524850519
0.4213304488847351
0.1365280484719059
0.4251097162894582
0.8595911731316150
0.5777780399453271
0.2047627501662884
0.4101426497191433
0.5811266883875064
0.0751880086165247
0.8617456814466556
0.1536592872909635
0.2886829922599975
0.1508053911122218
0.8613244247600459
0.2043897387855753
0.5957404227939725
0.4269132480394588
0.4232461827087615
0.9050418326654522
0.9056275857085205
0.1413272363791375
0.9074203860257329
0.9050491391850339
0.4529533357808937
0.2822729274590372
0.9058124199965637
0.4530320824373655
0.9072260965383889
0.2818217460739494
0.4530516101704253 


\begin{tabular}{|c|c|c|}
\hline & 21 & \\
\hline 789891677667145 & 1518404172801938 & 0.9299908068601519 \\
\hline 7510 & 194046370 & ร7000100000 \\
\hline 706703 & 099702825 & 57772 \\
\hline 478 & 154 & \\
\hline 242077447 & .5781999932907215 & .5756440924341062 \\
\hline 39 & & 51 \\
\hline 397 & 35 & 062 \\
\hline & & 329 \\
\hline 50 & & 62 \\
\hline 30 & 26 & 055 \\
\hline & 40 & \\
\hline 87 & 00 & \\
\hline & 306 & \\
\hline 99 & 65 & 307 \\
\hline 96763 & 393249 & \\
\hline & 52 & \\
\hline & & \\
\hline 4087550294563066 & 16 & \\
\hline 51 & 79 & 37 \\
\hline 0967 & 435 & 26 \\
\hline & 7 & \\
\hline & 0.86 & 241 \\
\hline 0.5760817426062470 & 0.4068119332736405 & 2158961476 \\
\hline & 0.9 & \\
\hline 51 & 046 & 871 \\
\hline 2817175104474805 & 0.9059246916751698 & 695 \\
\hline & 23 & 95 \\
\hline & 87 & 12 \\
\hline 2 & 0. & 69 \\
\hline 81 & 90 & 232 \\
\hline 9231835791419967 & & \\
\hline 61637577902628 & 0.089 & 0.2700 \\
\hline & 0.5410992095398214 & 539916592568 \\
\hline 0.0888680120791388 & 0.4475652123323129 & 0.4610746461604158 \\
\hline 170258909 & 0.9244892288188191 & 0.2231800101795920 \\
\hline 0.5414593417564946 & 0.4480870426774097 & 0.0439664154420447 \\
\hline
\end{tabular}




\begin{tabular}{|c|c|c|}
\hline & 74413 & \\
\hline 212182700140 & 0897546006919162 & 120070072 \\
\hline 0 & 474 & 2 \\
\hline 404014193053618 & .0889644952437394 & 537 \\
\hline 4 & 509 & 22 \\
\hline 30 & 446 & 76 \\
\hline & & 47 \\
\hline & & \\
\hline 5405256594269050 & 0.9244756 & \\
\hline 629 & 33 & \\
\hline 78826 & & \\
\hline$\$ 72579350001797$ & 19 & 0.04 \\
\hline & & \\
\hline 597 & 0.9 & \\
\hline 584 & 60963 & 3932 \\
\hline & & \\
\hline 063 & 0200550 & 38 \\
\hline 3 & 0.6 & 93 \\
\hline 6917123223606747 & 0.00425341354 & 38 \\
\hline & 78 & \\
\hline 62 & 0.0 & 396 \\
\hline 998069664389826 & .0047990685841865 & \\
\hline & & \\
\hline 8 & 2 & 69 \\
\hline 407 & 0.6 & 517 \\
\hline 21 & 0.3 & 0.0 \\
\hline 0030832304677903 & 0.0045048565338224 & 2716 \\
\hline 8 & 0.5 & 47 \\
\hline 715 & 0.006 & 1223 \\
\hline & & 0.0027555421991948 \\
\hline & 0.3 & 0064 \\
\hline 3987016371124104 & 0.006585502 & 0.294256371 \\
\hline 0.0059111232976192 & 0.58916954 & 0.00263 \\
\hline 363 & 0725520400 & 1387949013 \\
\hline 0.5883641269203038 & 0.0062410619937153 & 0.1994498446228699 \\
\hline 0.5882287370459842 & 0.3997235335587246 & 0.0032159848665902 \\
\hline 0.0055940815911819 & 0.0070070194156529 & 0.294306019922212 \\
\hline
\end{tabular}




\begin{tabular}{|c|c|c|}
\hline 633 & 85420395134 & \\
\hline 6931863008578 & 5888944309528029 & 00034383830384005 \\
\hline 6 & (20) & 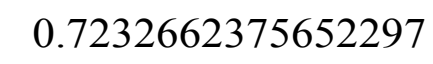 \\
\hline 407761363014759 & 77140 & 480 \\
\hline 66 & 209 & 350 \\
\hline 96 & 403 & 16 \\
\hline & & \\
\hline & & \\
\hline 0882259059415615 & .4470530816829973 & \\
\hline & 024 & \\
\hline & & \\
\hline 149 & 235 & 726 \\
\hline & & 08 \\
\hline & 0.5 & 999 \\
\hline 594 & 21321473 & 453 \\
\hline & & 51 \\
\hline & 04462700 & 87 \\
\hline 09 & 3 & 78 \\
\hline 0890028 & 0.4 & 77 \\
\hline 378 & & 50 \\
\hline 40415 & 0.0 & 88 \\
\hline 7995727 & .5400743 & 98669 \\
\hline & 98 & 366 \\
\hline 214 & 00 & 23 \\
\hline 130 & 0.92 & 500 \\
\hline 125 & 0.4 & 837 \\
\hline 3000305167042712 & 0.0026888446673855 & 816959 \\
\hline 50 & 0.2 & 59 \\
\hline 70570 & 0.691945846677 & 5481 \\
\hline & & 334896563926 \\
\hline 20 & 0.3 & 80 \\
\hline 0.6910836745967268 & 0.0045052167288778 & 0.6494271328592330 \\
\hline 0.299617425694 & 0.0049851250962536 & 0.8457349706656627 \\
\hline 5769 & 1327683279 & 2407 \\
\hline 0.0030942391152678 & 0.0039021317248634 & 0.6504451162591059 \\
\hline 0.2999071357439812 & 0.6925719868410811 & 0.5022385516894956 \\
\hline 0.6936978230941269 & 0.3042500983868727 & 0.502265606212025 \\
\hline
\end{tabular}




\begin{tabular}{|c|c|c|}
\hline 64 & 498 & \\
\hline 16 & 79195 & 33 \\
\hline 66 & 5206 & 2947 \\
\hline 52194 & 034 & 050704652 \\
\hline 30 & 332 & 082 \\
\hline 3978993918532737 & 0.0075412084882552 & 0.7936553395388052 \\
\hline 8 & & 0 \\
\hline 33 & 784 & 55 \\
\hline $7 \pi 0-10700$ & 0.0( & 946 \\
\hline & 55 & 146 \\
\hline 626 & 0.007 & \\
\hline & 708 & 478 \\
\hline & & \\
\hline 6074035 & 02 & \\
\hline 6 & 25 & 17 \\
\hline 21 & 42 & \\
\hline & 781 & \\
\hline & & \\
\hline 60 & 37 & 05 \\
\hline & 71 & \\
\hline & 0.9 & \\
\hline & 0.26229136 & 7761 \\
\hline 51 & 0.9 & 171 \\
\hline 79394 & 7003 & \\
\hline & & \\
\hline 83 & 01 & 02 \\
\hline 933897785127 & $0.1393 / 91000$ & 0.4626001202783410 \\
\hline & 0.2 & \\
\hline 2609397430903679 & 0.92684670613 & 0.36650922 \\
\hline 0.0795378087828922 & 0.2609812943954716 & 0.3664249665684201 \\
\hline 01 & 85 & 643 \\
\hline 0.2619987622258699 & 0.0785730560606696 & 0.4630236964766200 \\
\hline 0.924432871705 & 0.73 & 277 \\
\hline 096823026 & 0.732847 & 3542494 \\
\hline 0.9241588761290107 & 0.0794029707384702 & \\
\hline & 0.5 & 016584407 \\
\hline 0.7299921272327652 & 0.2606715968740402 & 0.465058455602147 \\
\hline
\end{tabular}




\begin{tabular}{|c|c|c|}
\hline 149 & 20831946789 & \\
\hline 325395896301947 & 2015556157053163 & 4666662430510867 \\
\hline 8 & 074 & 30 \\
\hline 9326791235881637 & .9326926114216495 & 665 \\
\hline 58 & 022 & 96 \\
\hline 268 & 857 & 00 \\
\hline 37506 & .0495124045445182 & 637 \\
\hline & & \\
\hline & 268 & \\
\hline & & \\
\hline 53005 & 040 & \\
\hline 396 & 665 & 225 \\
\hline & & \\
\hline & & \\
\hline 5434 & 92091622 & 468 \\
\hline & & 39 \\
\hline & 0265200 & 45 \\
\hline 4 & 59 & 06 \\
\hline 31 & 65 & 89 \\
\hline & 734 & 368 \\
\hline 84 & 725 & 93 \\
\hline 07015640260 & 6 & \\
\hline & 0 & \\
\hline 63 & 0.9 & 07 \\
\hline 80 & 184 & 326 \\
\hline 14 & 0.92 & 0.5 \\
\hline & 0.0793563587282400 & \\
\hline 3 & 0 . & 00 \\
\hline 797 & 0.73 & 0.5 \\
\hline 0.9253891786136627 & 0.079805013 & \\
\hline & 0.9 & \\
\hline 7322930130132320 & 0.2622650347071455 & 0.9630083716656958 \\
\hline 0.2014445494964322 & 0.9326451840077878 & 0.9665438783453117 \\
\hline 39 & 44 & 0.9 \\
\hline 0.9320392775136280 & 0.9324611274376376 & 0.6013212685249116 \\
\hline 0.9325915981728795 & 0.9330791134674429 & 0.9663978596667995 \\
\hline 0.8492447650428655 & 0.0498522947552629 & 0.525539366707011 \\
\hline
\end{tabular}




$\begin{array}{llll}0.0498726785657553 & 0.8504001064085682 & 0.5251018281592507 \\ 0.0494815401393975 & 0.0500448392625093 & 0.9257170550671135 \\ 0.0499264437675196 & 0.0496356518073506 & 0.5251564569291725 \\ 0.1186085358461933 & 0.8804747653944590 & 0.0600089517919157 \\ 0.8815102978838166 & 0.1184489559592399 & 0.4407998820310977 \\ 0.1202348108896716 & 0.1192211169564168 & 0.4404137834342610 \\ 0.8800584898098116 & 0.8805810568067312 & 0.0597083954780802 \\ 0.8800951155195466 & 0.1194951759620924 & 0.0598169648725561 \\ 0.1200447431986964 & 0.8800133762857274 & 0.4403728541046140 \\ 0.1194466338284113 & 0.8800896917318956 & 0.5601764341357680 \\ 0.8802461137395241 & 0.1198043678550266 & 0.9403867460170220 \\ 0.1191850262102607 & 0.1195812937412657 & 0.9404973256314711 \\ 0.8814646559071506 & 0.8793246728427901 & 0.5599925098606311 \\ 0.8814465584625357 & 0.1190966970764510 & 0.5599767670953864 \\ 0.1191934865092318 & 0.8807947214386491 & 0.9404094762058046 \\ 0.5294538887115580 & 0.2258748183810690 & 0.5094758532676995\end{array}$


The structure information of $1 \times 1 \times 2 \mathrm{UiO}-66$ primitive cell with one $\mathrm{K}$ at $\mathrm{K} 2^{\prime}$ site. UiO-66-K

1.00000000000000

$14.7785199142223291 \quad-0.0104287547755760 \quad 0.0060403739178511$

$\begin{array}{lll}7.3912039748059861 & 12.7958490781841849 & -0.0042778987663249\end{array}$

$14.7842810318728191 \quad 8.5073772304775090 \quad 24.1062915747572042$

$\mathrm{H} \quad \mathrm{C} \quad \mathrm{O} \quad \mathrm{Zr} \quad \mathrm{K}$

$\begin{array}{lllll}56 & 96 & 64 & 12 & 1\end{array}$

Direct
0.1322438008814918
0.5971127183287649
0.2127632558841392
0.5800458051556003
0.1509465176172199
0.4296319153161079
0.4089182640122934
0.8611399327945359
0.0757552495343501
0.8908602972181002
0.3845187413751318
0.2758603204199404
0.4082397466455264
0.1520068859257716
0.2894461557922735
0.8600087482586626
0.5800211251522659
0.0757492354444633
0.1505767946630311
0.4081618491451908
0.4305817661441616
0.5776852487129336
0.8624547900595944
0.2035403785469225
0.5814316560778328
0.4116119557366278
0.0748883205480472
0.1541227517955783
0.8643669540237091
0.2881353329112242
0.8595736849531622
0.1515548387569073
0.2050612606795028
0.3856544090044897
0.5579849043682777
0.4429039843249795
0.5788857546697733
0.1517645044993773
0.2041795779245193
0.1512076464075187
0.5785331342552004
0.4301845854456343
0.8603480758779685
0.4091770762366357
0.0758991262177442
0.4066757393949192
0.8626616577321640
0.2888918965130077
0.1329585426333112
0.4265754195683397
0.2976779442632344
0.5795594660729343
0.8612982328854324
0.0755116876376098
0.4093935731303461
0.1506308229398394
0.4301116180496187
0.8856994219933960
0.5588980133915789
0.1930113922135763
0.4109942595180095
0.5818449860039320
0.0749811729649108
0.8603617893561735
0.1516904103556637
0.2901563434391480
0.1529781828221021
0.8640366903046616
0.2032052014365226
0.5512857669994572
0.3840201449130644
0.4450737269363291
0.9056182551065600
0.9065358435437586
0.1413834161199058
0.9069128831719574
0.9052875917816477
0.4529912689422093
0.2823974545942901
0.9055123191098811
0.4534195456994515
0.9084012064523653
0.2807660209358120
0.4546869171356331 


\begin{tabular}{|c|c|c|}
\hline 84694778 & 30829286130208 & \\
\hline 799237433264545 & 1513246057938719 & 00708603415421800 \\
\hline 599 & 789 & \\
\hline 8606094819299439 & .4077763218 & \\
\hline 2 & 66 & 31 \\
\hline 52 & 81 & 90 \\
\hline 11495403482028 & 2056 & 1685 \\
\hline & & \\
\hline 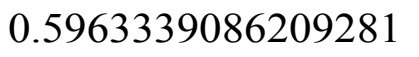 & 7001 & 157 \\
\hline & & \\
\hline 6795 & & \\
\hline 377 & 042 & 484 \\
\hline & & 51 \\
\hline & & \\
\hline 843 & 5670 & 885712053 \\
\hline & & \\
\hline & 70 & 0665 \\
\hline 6 & 4 & 26 \\
\hline 409467 & - & 977 \\
\hline & 66 & 608 \\
\hline 324 & 20 & 92 \\
\hline 3611939 & .15357379572 & 0895571 \\
\hline & & \\
\hline 38 & 3 & \\
\hline 97 & 0.9 & 918 \\
\hline 51 & 0.9 & 675 \\
\hline 0.2822902591612436 & 0.905925528499 & \\
\hline 4 & 0. & 21 \\
\hline 9021 & 64 & 177 \\
\hline 0.5416250133 & & \\
\hline & 0.9 & 683 \\
\hline 9392742679622560 & 0.4348414723769624 & 0.2634147126673925 \\
\hline 0.4461973893606627 & 0.0889822159094300 & 0.2703130706874842 \\
\hline 10 & $0 .$. & 0. \\
\hline 0.0875025775350411 & 0.4465080692379883 & 0.4621311371618857 \\
\hline 0.5399716444119207 & 0.9248880091485937 & 0.2230221061739687 \\
\hline 0.5420433358135859 & 0.4483667846664017 & 0.043983180659923 \\
\hline
\end{tabular}




\begin{tabular}{|c|c|c|}
\hline & 30757 & \\
\hline 9234721589720766 & .0892932619752140 & 0223702610 \\
\hline 49 & 5359 & 2 \\
\hline 5405225425741138 & & 494 \\
\hline & & 76 \\
\hline 15 & 548 & 83 \\
\hline 16086 & 209 & 8386 \\
\hline & & \\
\hline 7590961 & & \\
\hline & & \\
\hline 53 & & 6479 \\
\hline 4479068792114217 & .5424318 & 759 \\
\hline & & 00 \\
\hline 78 & & 50 \\
\hline 39350 & 339497 & 0.4692 \\
\hline & & \\
\hline 79316 & 0.3006717 & \\
\hline 08 & 0.6 & 60 \\
\hline 6923586921749236 & - & 65 \\
\hline 679 & 69 & 969 \\
\hline 36 & 6 & 52 \\
\hline 9094705304260 & 14 & \\
\hline 49 & & \\
\hline 78 & 0.0 & 45 \\
\hline 30 & 0.6 & 504 \\
\hline 34 & 0.30 & 508 \\
\hline & 0.0049439528 & \\
\hline 0 & 0 & 19 \\
\hline 749 & 0.00 & 0.00 \\
\hline 0.3999503595329567 & 0.0058274309846867 & \\
\hline 61 & & 391 \\
\hline 0.3985656849884408 & 0.0066098492441437 & 0.2943704213123119 \\
\hline 0.0052174429225585 & 0.5897194315382869 & 0.0029046731 \\
\hline 260 & 0.3 & 0. \\
\hline 0.5885165591271984 & 0.0063120570022229 & 0.1995329795382704 \\
\hline 0.5887373496276017 & 0.3995990143378996 & 0.0033783471760035 \\
\hline 0.0069053767483145 & 0.0076516586305810 & 0.294143099816261 \\
\hline
\end{tabular}




\begin{tabular}{|c|c|c|}
\hline 1 & 4 & \\
\hline 142 & 232 & 6 \\
\hline 22 & 7119487 & 519665995460 \\
\hline 91667 & 7770176 & 502853540049 \\
\hline 205 & 59 & 88 \\
\hline 9238791978979191 & .4459001601916253 & 0.7707854592781396 \\
\hline 47 & 0 & 36 \\
\hline 672230 & 0902 & 0.544 \\
\hline 79994725503387 & 147100271 & 912 \\
\hline 91 & 79 & 749 \\
\hline 3474 & 69 & 073 \\
\hline 222376 & & \\
\hline & & \\
\hline & & \\
\hline 1 & 9 & 16 \\
\hline & & \\
\hline & 0.446 & \\
\hline 5254 & 0.9236 & $0.7 / 0$ \\
\hline 0889840052644891 & 0.4459922150128821 & 07700 \\
\hline & 3 & \\
\hline & 0.0 & \\
\hline 50783 & 0.54001 & 0.7236956 \\
\hline 63 & 0.5 & 662 \\
\hline 90037 & 0.09006069 & 269309 \\
\hline & & \\
\hline 93 & 4 & 02 \\
\hline & $0.00550090 / 00$ & 0.00250020 \\
\hline & & \\
\hline 036877028102303 & 0.6908 & 0.65 \\
\hline 0.6924012906058962 & 0.0041442490475179 & 0.5017183916858722 \\
\hline 201 & 4 & 535 \\
\hline 0.6923173009963692 & 0.0033942606099496 & \\
\hline 69591415 & 0.00 & 0.84 \\
\hline 0.0039927685515792 & 0.6917139526 & 84419861267 \\
\hline 0.0042549068991751 & 0.0029901344701999 & 0.6504786310831319 \\
\hline 28314242885 & 0.6916676360770965 & 0.5022356082074371 \\
\hline 0.6904254608979650 & 0.3001290723342551 & 0.5021131867186006 \\
\hline
\end{tabular}




\begin{tabular}{|c|c|c|}
\hline 15 & 543653 & \\
\hline 682655350831 & 5880391437220837 & 0.6996874163231046 \\
\hline 59 & 269 & 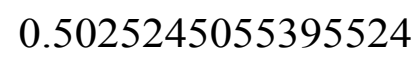 \\
\hline 4000245093744053 & .0053617623981648 & 86208668003 \\
\hline 3 & 34 & 06 \\
\hline 924 & 3575 & 3 \\
\hline 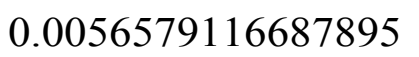 & 3 & 670 \\
\hline & & \\
\hline 8 & 28 & 75 \\
\hline & & \\
\hline 974 & & \\
\hline 352 & 385 & 021 \\
\hline & & 98 \\
\hline & & \\
\hline 71038 & 0.0799773275860780 & 534 \\
\hline & & \\
\hline 437989 & 0.2655706383755672 & 89 \\
\hline 2 & 08 & 86 \\
\hline 9250620558350153 & 47 & 61 \\
\hline & & 621 \\
\hline 50124 & 01 & 948 \\
\hline 7333962129761274 & .26303705 & 500015 \\
\hline & & \\
\hline 36 & 2 & 30 \\
\hline 604 & 0.732 & 774 \\
\hline 19 & 572 & 421 \\
\hline 0.0797212436279175 & 0.732845 & \\
\hline 3 & 0. & 56 \\
\hline 52506 & 0.92 & 875 \\
\hline 0.0797638159024937 & 0.2610003021017757 & 0.3662737700942926 \\
\hline & & 9026 \\
\hline 2619861366422103 & 0.0788325873135171 & 0.4632374506641536 \\
\hline 0.9257081389925851 & 0.7327610917679027 & 0.1308872460440971 \\
\hline & 0.7 & 0.0 \\
\hline 0.9275888618306898 & 0.0810841442434173 & 0.3663145981573928 \\
\hline 0.07838126546282 & 0.9257876898058602 & 0.1316243487026280 \\
\hline 0.7336973636666614 & 0.2649818607993594 & 0.4607209351272684 \\
\hline
\end{tabular}




\begin{tabular}{|c|c|c|}
\hline 36 & 247811426113 & \\
\hline 329868629505407 & 2008242922748069 & 4668372557520648 \\
\hline 53 & 571873856627 & 6 \\
\hline 334084236656037 & .9322262462941001 & 579 \\
\hline 0 & 43 & 53 \\
\hline 51 & 524 & 234 \\
\hline & & \\
\hline & & \\
\hline & 073 & \\
\hline 274 & 20 & \\
\hline 58946 & & \\
\hline 865 & 829 & 566 \\
\hline & & \\
\hline & & \\
\hline 464 & 0.2623636419 & 310 \\
\hline & & 422 \\
\hline & 50 & 02 \\
\hline 5 & 6 & 17 \\
\hline 209 & 49 & 93 \\
\hline 077 & 83 & 424 \\
\hline 36 & 0.0 & 12 \\
\hline 13 & 35106030 & .9630195794739923 \\
\hline & & \\
\hline 552 & 6 & 63 \\
\hline 923 & 43 & 0065 \\
\hline 502 & 0.9 & 816 \\
\hline 0.2623925133740151 & 0.0793949236 & \\
\hline 0 & 0. & 75 \\
\hline 2629538272484500 & 0.7334 & 0572 \\
\hline 9265687750634876 & & \\
\hline 50 & 0. & 16 \\
\hline 7323342815575701 & 0.2615571364333394 & 0.9634748996722733 \\
\hline 9900938033802 & 0.932644555 & 0.966675289 \\
\hline 28291488529075 & 0.2019845495 & 0.96661509 \\
\hline 0.9327093963314134 & 0.9316294166870805 & 0.6013189187626917 \\
\hline 0.9329570488881340 & 0.9330664924270148 & 0.9665004684481691 \\
\hline 0.8502250023178439 & 0.0494554301702071 & 0.525295796823817 \\
\hline
\end{tabular}




$\begin{array}{llll}0.0499245191802297 & 0.8503122356940948 & 0.5251163589803437 \\ 0.0498639237620845 & 0.0496222994457491 & 0.9260547812139650 \\ 0.0500091809726631 & 0.0490274263949781 & 0.5255921002340428 \\ 0.1192207675951780 & 0.8811226068038787 & 0.0599710203437088 \\ 0.8819472576669033 & 0.1171774898946183 & 0.4410425705450719 \\ 0.1203163127767868 & 0.1183965674231954 & 0.4410129725873178 \\ 0.8805219762291834 & 0.8810483314492978 & 0.0597454852579100 \\ 0.8805159511949654 & 0.1198078828020283 & 0.0599106654408540 \\ 0.1202318693522711 & 0.8794478305315447 & 0.4405831117926713 \\ 0.1198904571882254 & 0.8793393089382686 & 0.5602778842114824 \\ 0.8807397802777919 & 0.1196983453465579 & 0.9404482718803828 \\ 0.1197061834427132 & 0.1196357811935060 & 0.9405102309927806 \\ 0.8806443426511809 & 0.8793834457435938 & 0.5601573720481176 \\ 0.8808184257192847 & 0.1180143977423738 & 0.5605766677043628 \\ 0.1196835864173245 & 0.8805921395330908 & 0.9405142967571983 \\ 0.7424775442780919 & 0.4498076171160080 & 0.3713681856524300\end{array}$


The structure information of $1 \times 1 \times 2 \mathrm{UiO}-66$ primitive cell with one $\mathrm{K}$ at $\mathrm{K} 3$ site. UiO-66-K

1.00000000000000

\begin{tabular}{|c|c|c|c|c|c|}
\hline \multicolumn{4}{|c|}{14.7814833247843858} & -0.0039076970061537 & -0.0016712509779285 \\
\hline \multicolumn{4}{|c|}{7.3879369154374350} & 12.7946950777835546 & 0.0024904397687797 \\
\hline \multicolumn{4}{|c|}{14.7775566331635257} & 8.5338875921874671 & 24.1179370703782148 \\
\hline & $\mathrm{C}$ & $\mathrm{O}$ & $\mathrm{Zr}$ & $\mathrm{K}$ & \\
\hline 56 & 96 & 64 & 12 & 1 & \\
\hline
\end{tabular}

Direct
0.1521809791755145
0.5787729011640833
0.2037434545621601
0.5807823107963179
0.1487742574137575
0.4284805506349968
0.4084012647893536
0.8612847754872941
0.0756617541370153
0.8619560659630706
0.4076361634894514
0.2892679985308221
0.4080979680062152
0.1522968409767575
0.2891478093082085
0.8611212195310097
0.5794211963807584
0.0756465361506528
0.1514071688999658
0.4093949305119285
0.4299471467949588
0.5783384656858701
0.8612828058999858
0.2041932383171328
0.5794495267713017
0.4095611481934869
0.0753102885743306
0.1514849032142787
0.8613423425639869
0.2894845310212795
0.8613820898029422
0.1520656867299705
0.2039178895476186
0.4092838586759370
0.5812799582804623
0.4297320378021932
0.5784068818413652
0.1521550585711884
0.2039946079373701
0.1513446177900487
0.5797875790439908
0.4298671413131412
0.8608680011422297
0.4088499950809644
0.0758747363312820
0.4078503779258418
0.8614841031122754
0.2892852836003970
0.1527205019428687
0.4082140049468967
0.2889108683401914
0.5787685224452751
0.8613675955228075
0.0756009737691038
0.4083599077369681
0.1505391782366133
0.4301819969688171
0.8613436906850681
0.5783817595880023
0.2042005475664380
0.4090773863263790
0.5799777330118067
0.0752596921424768
0.8613734691728585
0.1527090572881503
0.2889445501935826
0.1515418978349008
0.8609943826982721
0.2043504519953001
0.5824326003673261
0.4147873389990842
0.4280376256911080
0.9054102020325033
0.9056168397363973
0.1412996602885101
0.9068179923339301
0.9063296064551104
0.4528115423992114
0.2819647700999225
0.9058121515898383
0.4531948313083303
0.9073767334674088
0.2818747893953679
0.4528605645339278 


\begin{tabular}{|c|c|c|}
\hline 001773240 & 527589760 & \\
\hline 789609123498090 & .1519714917630577 & 00300765800254783 \\
\hline 064 & 67 & 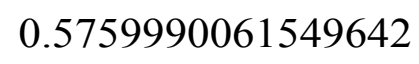 \\
\hline 8615512548369397 & 11539230 & 5037 \\
\hline 3 & 81 & 92 \\
\hline 81 & 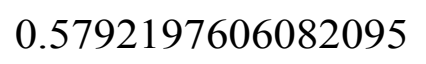 & 559 \\
\hline 33 & & 47 \\
\hline & & \\
\hline 07 & 00 & \\
\hline & & \\
\hline 318 & 53 & \\
\hline 805 & .5784497 & 820 \\
\hline & & 75 \\
\hline & & \\
\hline 92270 & .4087357834366424 & 0444 \\
\hline & & \\
\hline 518684292714741 & 0.4084077678836719 & \\
\hline 14 & 55 & 60 \\
\hline 122644 & 4 & 37 \\
\hline 89 & 526 & 16 \\
\hline 256 & 03 & 799 \\
\hline 8616745323309080 & .1528199951 & .7889494858405045 \\
\hline 72 & 3 & \\
\hline 579 & 07 & 93 \\
\hline 393 & 0.9 & 615 \\
\hline 44 & 0.906 & 52 \\
\hline & 0.9061958096258265 & \\
\hline 2 & 0. & 627 \\
\hline 7531 & 26 & 0.2 \\
\hline 0.5420145020571313 & 0.08642297 & \\
\hline 82 & 0.9 & \\
\hline 9249780898227239 & 0.4459040148150935 & 0.2701755227252448 \\
\hline 0.4461215935868807 & 0.0890508965294137 & 0.2701712293063037 \\
\hline 0.9 & $0 .$. & 0. \\
\hline 0.0884677099638857 & 0.4472393164369049 & 0.4616428573189749 \\
\hline 0.5402709483605036 & 0.9244660332520511 & 0.2232174608098133 \\
\hline 0.5409983613623334 & 0.4473549734557358 & 0.043905549387093 \\
\hline
\end{tabular}




\begin{tabular}{|c|c|c|}
\hline 2 & & \\
\hline 5654775 & .0890090018840980 & 02230507603 \\
\hline 478455101479224 & 164 & 093 \\
\hline 5943 & 355 & .2231157710477179 \\
\hline 327 & 612 & \\
\hline 239603822823715 & .4470029816744784 & 0.0442437580607142 \\
\hline 72 & 90 & 85 \\
\hline 13 & 637 & \\
\hline & & \\
\hline 594 & & \\
\hline 356 & 97 & \\
\hline & & \\
\hline & & \\
\hline & 0.9243250 & 078 \\
\hline 99 & 16 & 546 \\
\hline 97796058391458 & 0.004509561 & 0.001 \\
\hline 72 & 929 & 0.34 \\
\hline & & \\
\hline 091 & 368 & \\
\hline 6 & 07 & 46 \\
\hline & 91 & \\
\hline & 657 & \\
\hline 0039219117137425 & 0.69 & 0.0 \\
\hline 0.0034162337594161 & 0.0034570594905594 & 0.1503519 \\
\hline & & \\
\hline 342 & 16 & 0.00 \\
\hline 0035985686677711 & 0.0044713429174167 & 0.34593145 \\
\hline 44 & 0.5 & 0 . \\
\hline & 0.0 & \\
\hline 76 & 0. & 0 . \\
\hline 34 & 72 & 545 \\
\hline 0.3987842351641526 & 0.0064486252989269 & \\
\hline 0.0058741641462518 & 0.5892318296 & 0.0026 \\
\hline 055125418762878 & 0.3998329397114347 & 0.0029027062787266 \\
\hline 0.5884031195107912 & 0.0062585701327881 & 0.1995493150120849 \\
\hline 0.5884772502721528 & 0.3996693479259073 & 0.00283732214110 \\
\hline 0.0058868149377011 & 0.0065323574786063 & 0.2943187060375524 \\
\hline
\end{tabular}




\begin{tabular}{|c|c|c|}
\hline 84 & 3712 & 0 . \\
\hline 2379940038966 & 5800807 & 00027 \\
\hline 531 & 7388040281759 & 1164 \\
\hline 7649732140545 & 0.0888859201162928 & 0.9616824417621216 \\
\hline 907 & 725 & 883 \\
\hline 9245957279128367 & 0.4460543036875403 & 0.7701951343059439 \\
\hline 9951 & 78 & 78 \\
\hline 9241551739599327 & 0.54110250421 & 0.0454 \\
\hline 0880341488468564 & $0.4470 s$ & 115 \\
\hline 15 & 0.9 & 0.7 \\
\hline 11 & 0.4517 & 0.5 \\
\hline 523 & 949 & 0.7 \\
\hline & & \\
\hline 0.4459949456722071 & 0.5406321675 & 0.9 \\
\hline 73 & 16 & 55 \\
\hline 447968 & 0.54 & \\
\hline 9242709619542946 & 0.4469286637424911 & 0.5440 \\
\hline 83 & 0.9 & 0. \\
\hline 0888804696223545 & 0.4463310222185269 & 0.77014122 \\
\hline 678 & 0.92166 & 0.54 \\
\hline 4466399353223184 & 0.0 & 0.9 \\
\hline 9242824811474996 & 0.5404542545014448 & 0.72320 \\
\hline 54 & 0.5 & \\
\hline 561 & 0.08 & 0.7 \\
\hline 671 & 0.92 & 0.7 \\
\hline 27422416 & 0.44 & 0.9 \\
\hline 2996501625854853 & 0.0034321878971294 & 0.5024694690849267 \\
\hline 66 & 0.3 & 0 . \\
\hline 953 & 0.6920 & 0.6 \\
\hline 0.6933838632197628 & 0.0009523655057935 & \\
\hline 18 & 0.30 & \\
\hline 0.6931301779771022 & 0.0008288138414028 & 0.6526296141614043 \\
\hline 0.2990609233127993 & 0.0048524981692289 & 0.8462665902429 \\
\hline 53 & 0.6 & 0. \\
\hline 0.0032157721213584 & 0.0038715943772189 & 0.6503665206855179 \\
\hline 0.2992351020414686 & 0.6922669370115856 & 0.5022773174738103 \\
\hline .6942720837768862 & 0.3066002433942066 & 0.5000871402946034 \\
\hline
\end{tabular}




\begin{tabular}{|c|c|c|}
\hline & 52 & \\
\hline 058426215538544 & .5888490369539567 & 060 \\
\hline 913175501827269 & .0030343117209322 & 0.50 \\
\hline 340 & 29188 & 5003 \\
\hline 728 & 2169 & \\
\hline 3983895342618033 & 1 & 772112633 \\
\hline 22 & 59 & \\
\hline 63 & 4733 & \\
\hline & & 99 \\
\hline 7 & 328 & \\
\hline 31 & 944 & \\
\hline & & \\
\hline 49 & & \\
\hline 42 & & \\
\hline 592 & 921 & $0.40<0$ \\
\hline Conornous & 0.9266757134066249 & 0.039 \\
\hline & 649 & \\
\hline & & 177 \\
\hline 9257341524942359 & 072286 & 3950 \\
\hline 39 & 4 & 86 \\
\hline & 996 & 929 \\
\hline & 6 & \\
\hline 009 & 0.92 & 988 \\
\hline 2496609 & 0.0787933519769 & 937 \\
\hline & & \\
\hline 7322287417850469 & 160 & 442 \\
\hline 0792580574805649 & 015317 & 0.4626 \\
\hline & 69 & 078 \\
\hline 7771157 & 0.9 & \\
\hline 97 & 0.2 & 270 \\
\hline 2109 & 425 & 540 \\
\hline 2612588101357465 & 0.0789391769604702 & \\
\hline 0.9255926142376667 & 0.7326701848440460 & 0.1309096 \\
\hline 75648511 & 0.7331690320071845 & 0.0396168170 \\
\hline 0.9252715009340159 & 0.0800583728682830 & 0.3665536621115965 \\
\hline 0.0787550 & 12656 म & 0.1313220225953 \\
\hline 0.7328212541207230 & 0.2639601958669260 & 0.4624919059242477 \\
\hline
\end{tabular}




\begin{tabular}{|c|c|c|}
\hline 06632 & & \\
\hline 10 & 103 & 0 \\
\hline 321669409791408 & .9324076411937133 & 101140000 \\
\hline 4 & 6 & 93 \\
\hline 76523 & 60824 & 416 \\
\hline 7546338 & & \\
\hline & & \\
\hline 25 & 53 & \\
\hline & & \\
\hline & & \\
\hline & 71 & \\
\hline 5321 & 年 & 89 \\
\hline 610461729698366 & 0.0806584086825551 & \\
\hline 790 & 66257 & 886 \\
\hline & & \\
\hline 60075 & 0.9250 & 366 \\
\hline 3 & 636 & 93 \\
\hline & 35 & 98 \\
\hline & & 68 \\
\hline 78 & 0.73 & 67 \\
\hline 7317879580097293 & 0.0796664928004874 & 0.631739139 \\
\hline 37 & 0.7 & \\
\hline 51 & 42 & 48 \\
\hline 2604794898404062 & 62895 & 33 \\
\hline & 40 & 541 \\
\hline 738446507 & 0.92 & 50 \\
\hline 6 & 0. & 864 \\
\hline 412 & 0.733 & 143 \\
\hline & 0.7329995922861429 & \\
\hline 1 & 39 & 0 . \\
\hline 782864999898830 & 0.9253771106786832 & 0.6313294612917396 \\
\hline 0.7323829978307015 & 0.2618827571981828 & 0.9629697705649618 \\
\hline & 0.933 & 5310 \\
\hline .9319949497885184 & 0.2024302391347098 & 0.9664910344670044 \\
\hline 0.9319752223068470 & 0.9331627350753975 & 0.6007996487530118 \\
\hline 63 & 0.9 & 0.9 \\
\hline 0.8445587755943331 & 0.0521154788490119 & 0.5259894886883658 \\
\hline
\end{tabular}




$\begin{array}{llll}0.0493227197276204 & 0.8509983915554895 & 0.5249694955900189 \\ 0.0492928785644704 & 0.0501449689153987 & 0.9257805298334686 \\ 0.0494818201387028 & 0.0498016408010742 & 0.5251211899813771 \\ 0.1190880392161540 & 0.8806583854793396 & 0.0598157084616099 \\ 0.8818719329920712 & 0.1197678598133551 & 0.4398022492152525 \\ 0.1204796973448071 & 0.1196352664264139 & 0.4402325756115050 \\ 0.8802670978109968 & 0.8806530651777088 & 0.0597646413496858 \\ 0.8801395910047932 & 0.1195271397438748 & 0.0598710524961617 \\ 0.1200714856793243 & 0.8801966101474333 & 0.4401310918281960 \\ 0.1198381189174790 & 0.8800297508044315 & 0.5600527436530398 \\ 0.8802295209721899 & 0.1197905233627918 & 0.9403883346653125 \\ 0.1188326724719106 & 0.1199317684133989 & 0.9405767049232064 \\ 0.8818366910868621 & 0.8795197882100143 & 0.5596417075868114 \\ 0.8819401915366837 & 0.1196018432809468 & 0.5596928015829583 \\ 0.1189597488496417 & 0.8809015253539489 & 0.9404393857564989 \\ 0.5981148715926109 & 0.1358038660836552 & 0.5662254107610144\end{array}$


The structure information of $1 \times 1 \times 2 \mathrm{UiO}-66$ primitive cell when all sites are occupied by K

UiO-66-K

1.00000000000000

$\begin{array}{rrr}15.5514549769461450 & -0.0039218334533265 & 0.0037682822142754 \\ 7.7739154310646175 & 13.4662605738681922 & 0.0043485678622241 \\ 15.5616552920577238 & 8.9824959513777998 & 25.2963802442744203\end{array}$

$\mathrm{H} \quad \mathrm{C} \quad \mathrm{O} \quad \mathrm{Zr} \quad \mathrm{K}$

$\begin{array}{lllll}56 & 96 & 64 & 12 & 80\end{array}$

Direct
0.1313236940385713
0.5823107287792392
0.2181759428576856
0.5829771774122624
0.1321939903613786
0.4236803387966528
0.4367169567077130
0.8500246939825705
0.0657892981792756
0.8493318200145348
0.4364468344421855
0.2911881806954971
0.4363908172096060
0.1317526725153175
0.2909555058336832
0.8490024732506614
0.5822663160958935
0.0657040916060896
0.1320228428897497
0.4368057945948292
0.4244150405230555
0.5817690326988104
0.8496826622409922
0.2183409144606014
0.5819641799412114
0.4368249454241814
0.0657940373952947
0.1320641443042871
0.8500303173257577
0.2907677090870900
0.8496850849862790
0.1316933152102346
0.2183949523548838
0.4373120221710363
0.5826339672307108
0.4244181088056510
0.5819127045334771
0.1316528834257377
0.2183295630568789
0.1322086079568386
0.5820954325100650
0.4243866785609661
0.8486898150578982
0.4370609061528873
0.0657724904969122
0.4363190561092846
0.8495201476846472
0.2910715644397834
0.1318205256520448
0.4365992686851825
0.2907542944758201
0.5821686629352704
0.8501261105321278
0.0655012144708888
0.4372796017124411
0.1321402668915312
0.4240065212037066
0.8491460850308228
0.5820526159930686
0.2184979367859188
0.4368799511562113
0.5820583244040649
0.0658292609424624
0.8499967112811095
0.1319236293847298
0.2908868579387844
0.1315675071954904
0.8497398892611761
0.2183632971628056
0.5827755097089962
0.4376088802554071
0.4243124953523863
0.9088084928937923
0.9091783635048334
0.1363566498880526
0.9101560816748320
0.9099056696810431
0.4547707641999654
0.2708090889120581
0.9098920374460160
0.4548987227849394 


\begin{tabular}{|c|c|c|}
\hline 96 & & \\
\hline 734 & 991 & 5مو \\
\hline 5826640712968466 & .1323931772146251 & 081 \\
\hline 324 & 481 & 358 \\
\hline 302 & 536 & 555 \\
\hline 115974649 & 245 & \\
\hline & 04 & \\
\hline 527 & 560 & 34 \\
\hline 96 & 97 & \\
\hline 67 & & \\
\hline 1 & 76 & \\
\hline 95303972187360 & 71 & 13 \\
\hline 367062765640392 & 0.58235511 & 973 \\
\hline 3129 & 52177411 & 623 \\
\hline & & \\
\hline 632 & 0.4369 & \\
\hline 8 & 72 & 417 \\
\hline & & \\
\hline & 76 & \\
\hline 43 & 0.1 & 75 \\
\hline 3800211495148 & 79332753691 & 0.7183782717262825 \\
\hline & 4 & 14 \\
\hline 58 & 85 & 784 \\
\hline & 0.8497 & 84 \\
\hline & & \\
\hline 7 & 0.9 & 78 \\
\hline 4 & 0.9 & 0. \\
\hline 536 & 0.90 & 158 \\
\hline & 0.2709899995815189 & \\
\hline 8 & 0.5 & 0 . \\
\hline 496682223821392 & 0.074141523 & 5021 \\
\hline 0.4585953131179094 & 0.9196039241773298 & \\
\hline 928292 & 0.4583099132 & 5110 \\
\hline 0.4582702374965621 & 0.0730338852777369 & 0.2746594721069702 \\
\hline 0.9185489074923312 & 0.5494816499159284 & 0.0363575747871682 \\
\hline & T. & 0.4 \\
\hline 0.5489459015863604 & 0.9193412053559724 & 0.2291136748781273 \\
\hline
\end{tabular}




\begin{tabular}{|c|c|c|}
\hline & & \\
\hline 4 & 4 & \\
\hline 9 & 1978070711 & \\
\hline & & \\
\hline & & \\
\hline & 26 & \\
\hline & & \\
\hline & & \\
\hline & & \\
\hline & & \\
\hline & & \\
\hline & & \\
\hline & & \\
\hline & & \\
\hline & & \\
\hline & & \\
\hline & \multicolumn{2}{|c|}{$0.0003927514577293-0.0001857125239330$} \\
\hline & & \\
\hline & & \\
\hline & \multicolumn{2}{|c|}{$0.0002616782254785-0.0004226919767410$} \\
\hline 4 & \multicolumn{2}{|c|}{$0.3082960134109825-0.0003119592754837$} \\
\hline & & \\
\hline 0 & & \\
\hline & \multicolumn{2}{|c|}{$0.6923318826778494-0.0002691439608895$} \\
\hline & & \\
\hline & 0.692 & 0 \\
\hline 4 & \multicolumn{2}{|c|}{$0.3083274643895323-0.0002916$} \\
\hline 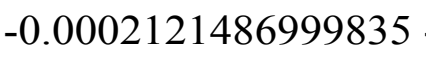 & 556 & 0.3 \\
\hline .002817047 & 601961874448 & 0.2 \\
\hline \multicolumn{3}{|c|}{$0.6015693287535047-0.0014901690597020-0.0017513452306246$} \\
\hline \multicolumn{3}{|c|}{$0.4032303577892232-0.0016885343320549-0.0015010900701520$} \\
\hline 0.0024775525180019 & 0.4028892083721184 & \\
\hline 0.402701 & 0023 & 0.3 \\
\hline $261+2+2$ & 0.60 & 0 \\
\hline-0.0026981954309752 & 2262721246 & $0 \Omega$ \\
\hline & 75265070527 & \\
\hline .6013092034256460 & 0.403392865335832 & 0.001369 \\
\hline
\end{tabular}




\begin{tabular}{|c|c|c|}
\hline \multicolumn{2}{|c|}{$-0.0020289391037273-0.0020783971559685$} & 0.3007528306939432 \\
\hline \multicolumn{2}{|c|}{$-0.0025149434175845-0.0024263533652349$} & 0.2015171117899778 \\
\hline 0.4031748581809975 & 0.6015595946170464 & -0.001293480300 \\
\hline 0.0730368782945578 & 0.5492633614950596 & 0.7289768478355788 \\
\hline 0.5494103557790846 & 0.0742888713072705 & 0.9588238807693535 \\
\hline 0.4587460735325217 & 0.9195771861381802 & 0.5364660319132749 \\
\hline 0.9189362443022893 & 0.4582286765857172 & 0.7747021039316997 \\
\hline 0.4583884461073332 & 0.0728508761706995 & 0.7746628 \\
\hline 0.9185924433403417 & 0.5495085351722059 & 0.5363796303008291 \\
\hline 0.0732838452979620 & 0.4584880076319861 & 0.9592580866219833 \\
\hline 0987733798 & 41213579 & 0.72909 \\
\hline 0.5494167047790188 & 0.4587628950640664 & 0.5366593056078593 \\
\hline 0.0733036117802896 & 0.9195565044301146 & 0.7745050372692820 \\
\hline 0.9193207158795133 & 0.0732209189468548 & 0.7291161946116356 \\
\hline 0.4582494230968034 & 0.5492898344727699 & 0.9593567755517906 \\
\hline 0.5492542198334256 & 0.0729689684403757 & 0.7291147798952349 \\
\hline 0.0733933944386087 & 0.5493010990468583 & 0.9591951215213720 \\
\hline 0.9185879434899263 & 0.4587351913650956 & 0.536475735 \\
\hline 0.4581453965153013 & 0.9189400066368952 & 0.7747010202196487 \\
\hline 0.0729530442912540 & 0.4584053900881848 & 0.7745461009403281 \\
\hline 453855929 & 0.919 & 0.5362 \\
\hline 0.4585685715933310 & 0.0740322942442707 & 0.9 \\
\hline 0.9191199208503703 & 0.5490294445926065 & 0.7290783918719578 \\
\hline 0.4587647630888697 & 0.5494760978514054 & 0.5366482368938312 \\
\hline 0.9192886061654375 & 0.0731767725071850 & 0.7746012289078977 \\
\hline 0.0732 & 0.9 & 03136 \\
\hline 0.5490402982878776 & 0.4585304147066419 & 0.9593035149993367 \\
\hline 0.3080736921704074 & 0.0003361984147970 & 0.4999423365256888 \\
\hline-0.0003752186571013 & 0.3078033037139833 & 0.8464993159964662 \\
\hline-0.0012261226195996 & 0.6936673755320203 & 0.6541094496331359 \\
\hline 0.6925045944168158 & 0.0002662539733727 & 0.4995811866239884 \\
\hline-0.0004786772573230 & 0.3082836346874263 & 0.4997302157150968 \\
\hline 0.6934570773592250 & -0.0009798949645060 & 0.6541264056903950 \\
\hline 0.3076732954614326 & -0.0002717821340796 & 0.8464880381138189 \\
\hline-0.0003324011189130 & 0.6923582421613039 & 0.4997421378908981 \\
\hline-0.0011633147402766 & .0010850665715008 & 0.6542363921948158 \\
\hline 0.3082205543401633 & 0.6925525441139434 & 0.499922943740551 \\
\hline
\end{tabular}




\begin{tabular}{|c|c|c|}
\hline & & \\
\hline-005227205 & $0=$ & 0816 \\
\hline & & \\
\hline 468 & 59 & 049 \\
\hline 0 & & \\
\hline 91 & 513023566 & 229 \\
\hline & & \\
\hline 58 & & \\
\hline & & \\
\hline & & \\
\hline & & 40 \\
\hline 0 & & \\
\hline & & \\
\hline & & \\
\hline 12 & 748 & \\
\hline & 0.07 & \\
\hline & & \\
\hline & & \\
\hline & 5 & \\
\hline & & \\
\hline & & \\
\hline & & \\
\hline & & \\
\hline & & \\
\hline & & \\
\hline & & \\
\hline 8 & 33 & 27 \\
\hline & & \\
\hline & & \\
\hline 22 & 77 & 51 \\
\hline 93 & 26 & 32 \\
\hline & & \\
\hline 99 & 0.0 & \\
\hline & & דים \\
\hline & & \\
\hline & & \\
\hline 0.0774610226730597 & 0.928717909522427 & 0.129996465334825 \\
\hline
\end{tabular}



0.7327999736173684
0.2609100700604415
0.4644066628047217
0.1933010760082821
0.9356476078309476
0.9354675797220392
0.1934189010299127
0.4679302606679139
0.9347187197313880
0.9349754160900100
0.4676485171858589
0.9356467591487397
0.9356344459398367
0.0974222949847397
0.8534635784277047
0.0487114061509762
0.4679059907881417
0.0483666878312455
0.8537457695872580
0.0246440882815737
0.0491352227291018
0.0491343388282284
0.0246619140422083
0.0483480540484167
0.0489093743000799
0.4264682008491131
0.0771864948927995
0.7339455015895898
0.0245238595417269
0.7329206972360468
0.0781704236525299
0.6300800215983468
0.2602702109443985
0.9297628680212389
0.9289257481627071
0.2597040251765513
0.9642270134447855
0.2601374475777354
0.0778602371544658
0.5390685474983006
0.9286753005174708
0.7327092796886689
0.8669696504659905
0.0777818809547402
0.2607269102099703
0.8666967156888215
0.7333131771584043
0.9290581793403194
0.5387371063359726
0.7329924444500480
0.2607362565883420
0.9643403610691595
0.0776065849956732
0.9288847782985055
0.6297929463208989
0.9285960040712102
0.0775082613214690
0.5388168592021045
0.2604207787054961
0.7327019610419443
0.8668276151138506
0.7336960611810122
0.0774264832183646
0.6300174547663187
0.0777564766396032
0.7325304827854353
0.9645095694440438
0.9284287699311968
0.2608872868414226
0.6301274870196689
0.2596235048562225
0.9289707917919551
0.9643938222130455
0.0778226582421122
0.2601645352371302
0.5386042815684827
0.7326972566442490
0.9291743354445913
0.8670198147504307
0.2606093147933450
0.0781436142367966
0.8666897220167243
0.9288160942651892
0.7334751250657758
0.5386091775953059
0.2607620773334920
0.7330294125698287
0.9642697929738784
0.9288966144905150
0.0776660981111768
0.6297726374372027
0.0774567272124821
0.9287309106868673
0.5388639899625214
0.7325486792866098
0.2607623873146664
0.8668037262430519
0.1931788995009988
0.9356652040502288
0.6299825591246453
0.9643247417833926
0.9353954689261389
0.1934730001046312
0.9678868244269792
0.9348060753704931
0.9348872783880745
0.9676599333135100
0.9355793508065908
0.9357288349258697
0.5974364348530447
0.9678629039731866 


\begin{tabular}{|c|c|c|}
\hline 16 & 6 & \\
\hline 53 & 40 & 86 \\
\hline 50 & 369044987659 & 301 \\
\hline 5142279 & 999625761347 & .5245250610704243 \\
\hline 50 & & \\
\hline 226 & 540 & 01 \\
\hline & & 82 \\
\hline 09 & & \\
\hline 3840102450462231 & 700 & \\
\hline 32 & 06 & 50 \\
\hline & & \\
\hline 26 & 405 & 5083 \\
\hline & & \\
\hline 867 & 0.8 & \\
\hline 6 & 66 & 375 \\
\hline & & \\
\hline & 250 & \\
\hline 6 & 26 & \\
\hline 1196785777993746 & 55 & \\
\hline 88 & 20 & 070 \\
\hline 3676 & 1473723 & 031 \\
\hline 714803914792079 & .2714285959625423 & 160482273 \\
\hline & & \\
\hline 36 & 11 & \\
\hline 94 & 0.4 & 35 \\
\hline 5607 & 67724 & 940 \\
\hline 665635010974098 & 0.4290815993379929 & \\
\hline & 0 & 34 \\
\hline 068 & 16 & 217 \\
\hline 0.7662516871197521 & 0.766330514 & \\
\hline & 577 & 6143 \\
\hline 2714515702383026 & 0.5745599163673798 & 0.4414698130137791 \\
\hline 0.2708421462072217 & 0.5732552337799123 & 0.6355023262510430 \\
\hline & 20 & 0.5195738902 \\
\hline 0.4280932300452155 & 0.7674322462671912 & 0.3833582904452624 \\
\hline 0.2705338 & 0.8852700170403927 & 0.2867519086441870 \\
\hline .6239234726126009 & 0.8763049653304745 & 0.3117613711567379 \\
\hline
\end{tabular}




\begin{tabular}{|c|c|c|}
\hline 84880 & 130060 & \\
\hline 710201053011610 & 8849049515930371 & 0.6357204591796648 \\
\hline 34 & 717792 & 0 \\
\hline 4278063927643165 & & \\
\hline 8 & 56 & 24 \\
\hline 05 & 986 & 846 \\
\hline 9607 & 10 & 842 \\
\hline & & \\
\hline 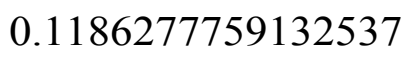 & & \\
\hline & & \\
\hline 9023 & & \\
\hline 5879 & 177 & 970 \\
\hline & & 89 \\
\hline & & \\
\hline 5887 & 495 & 930 \\
\hline & & \\
\hline 137 & 0885620 & 14 \\
\hline 21 & 5 & 38 \\
\hline 882869212575 & 0.271540217 & 53 \\
\hline 5063 & 0.03 & \\
\hline 18 & 0.3 & 376 \\
\hline 5 & 6 & 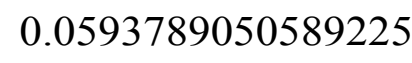 \\
\hline & & \\
\hline 504 & 0.0 & 269 \\
\hline 34 & 625 & 943 \\
\hline 450 & 0.6 & 438 \\
\hline & 0.38 & 23862 \\
\hline 6 & & 915 \\
\hline 95 & 0.1 & 0. \\
\hline & 0.6240 & \\
\hline & & 9744 \\
\hline 2712503846499483 & 0.8837480770994888 & 0.1357253155409106 \\
\hline 0.1192507707559423 & 0.3802758837892753 & 0.6902824087766465 \\
\hline 27 & 0 & 0 . \\
\hline 0.7662062088188276 & 0.0385872897592601 & 0.7145094061845324 \\
\hline 0.7678907154632847 & 0.0373719402135522 & 0.38322405940205 \\
\hline .2706963384729658 & 0.5744251578205923 & 0.9417798095648454 \\
\hline
\end{tabular}




$\begin{array}{llll}0.8848629120950244 & 0.2709261890035454 & 0.2866264003608604 \\ 0.8823811583032780 & 0.2715345870289680 & 0.6358453079073773 \\ 0.0388880323120711 & 0.7662879706299901 & 0.2143927828615919 \\ 0.6417816732739535 & 0.1193643259207620 & 0.5599016517063593 \\ 0.1187056780761596 & 0.6420953762109524 & 0.5599674656556743 \\ 0.6236272837129365 & 0.6245222924058055 & 0.9371649323624297 \\ 0.1190677131425411 & 0.3807034696531865 & 0.1901883390569609 \\ 0.8761963636119120 & 0.6241986400327953 & 0.3117547250980662 \\ 0.1186435063057011 & 0.3807721370278586 & 0.5598837244228402 \\ 0.6236875846972629 & 0.8775570900664648 & 0.4371393286631150 \\ 0.0385405085529851 & 0.4285080988779068 & 0.8829581449459940 \\ 0.0388239507581692 & 0.7667596333037273 & 0.3829832949403122 \\ 0.8758067853385491 & 0.8763556096930165 & 0.3117844514009930 \\ 0.8763863831578018 & 0.8757219492287475 & 0.8117867041650852 \\ 0.6238764505087319 & 0.8777551949500872 & 0.9369548605129761 \\ 0.8773175074456768 & 0.6241953159911244 & 0.9369629286070971 \\ 0.6417194451106524 & 0.1191685467090364 & 0.0600287454504675 \\ 0.1185829252261497 & 0.6420808003869626 & 0.0601000346394419 \\ 0.1182500393711813 & 0.1194606896240732 & 0.5600373947277438 \\ 0.1196726310231052 & 0.1197943663221633 & 0.3205895021179693 \\ 0.1196614133491806 & 0.1199292786973923 & 0.8205891886240578 \\ 0.1183308584301888 & 0.1193449674508474 & 0.0600340796342538\end{array}$

